\title{
Réflexions sur l'articulation entre didactique et épistémologie dans le domaine des grandeurs et mesures dans l'enseignement primaire et secondaire
}

\author{
Valérie Munier et Dominique Passelaigue
}

URL : http://journals.openedition.org/trema/2840

DOI : 10.4000/trema.2840

ISSN : 2107-0997

\section{Éditeur}

Faculté d'Éducation de l'université de Montpellier

\section{Édition imprimée}

Date de publication : 1 décembre 2012

Pagination : 106-147

ISBN : 1167-315X

ISSN : 1167-315X

\section{Référence électronique}

Valérie Munier et Dominique Passelaigue, «Réflexions sur l'articulation entre didactique et épistémologie dans le domaine des grandeurs et mesures dans l'enseignement primaire et secondaire », Tréma [En ligne], 38 | 2012, mis en ligne le 01 décembre 2014, consulté le 30 avril 2019. URL : http://journals.openedition.org/trema/2840; DOI : 10.4000/trema.2840 


\title{
Réflexions sur l'articulation entre didactique et épistémologie dans le domaine des grandeurs et mesures dans l'enseignement primaire et secondaire
}

\author{
Valérie Munier et Dominique Passelaigue
}

1 Cet article s'appuie sur des travaux menés depuis plusieurs années en collaboration avec différents chercheurs sur les grandeurs et mesures (Jean-Michel Dusseau, Jean-François Favrat, Françoise Langlois, Hélène Merle et Mohamed Robert) et reprend certains des résultats de ces travaux pour les mettre en perspective $\mathrm{du}$ point de vue des articulations entre épistémologie et didactique.

\section{Introduction}

2 L'importance de la mesure pour le scientifique est indéniable, et pour Ullmo, c'est même un lieu commun de dire que

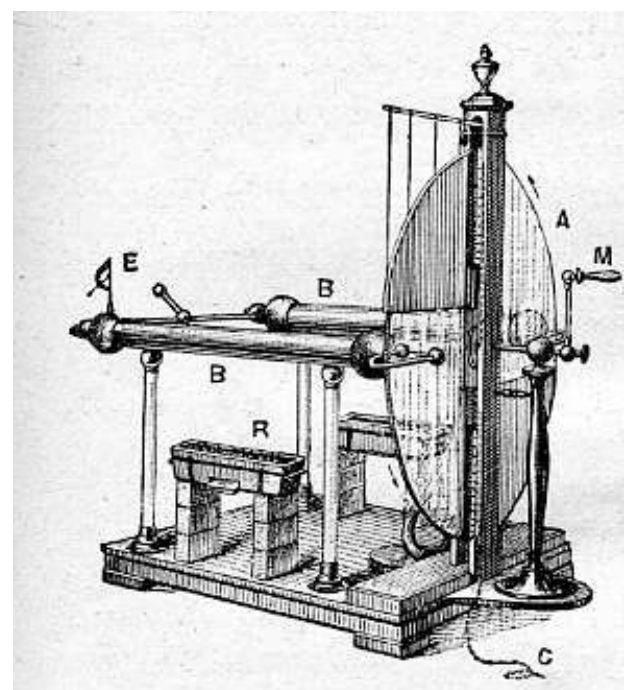

Fig. 208. - Machine de Ramsden. (Yue d'ensemble.) «la Science porte sur la quantité et s'exerce au moyen de la mesure » (Ullmo, 1969, p 23). De nos jours, il est ainsi admis que la science ne peut se faire sans activité de mesurage, c'est-à-dire sans la présence d'un « ensemble d'opérations ayant pour but de déterminer une valeur d'une grandeur» (Bureau International des Poids et Mesure, noté dans la suite BIPM, 2008). Les concepts de 
grandeur et mesure sont, de ce fait, au cœur même de l'activité du physicien. De la même manière, les concepts de grandeur et mesure sont essentiels dans l'enseignement :

Elles [les grandeurs] demeurent sans doute un passage obligé pour les enfants. D'abord nous vivons au milieu d'objets qu'il nous faut, avant toute idée élaborée de mesure, saisir sous l'aspect de grandeurs [...]. Ensuite, puisqu'on recourt sans cesse à des mesures dans la vie civilisée d'aujourd'hui, il faut bien apprendre en quoi elles consistent et ce qu'elles nous apportent [...] (Friedelmayer, 2001).

3 La question des grandeurs et de la mesure est abordée à l'école dès le plus jeune âge. L'enseignement des grandeurs et de la mesure concerne donc l'ensemble des enseignants, de la maternelle à l'université. Dans cet article, nous nous intéressons à l'enseignement des grandeurs et mesures depuis l'école élémentaire jusqu'au lycée, en France, et plus particulièrement à la question de l'articulation entre didactique et épistémologie dans ce domaine. Il s'agit à la fois de chercher à identifier les positions épistémologiques des concepteurs de programmes et des enseignants sur ces questions et de discuter l'influence de ces postures épistémologiques sur les contenus et les modes d'enseignement préconisés par les instructions officielles aux différents niveaux. Nous questionnons en outre l'intérêt d'une approche épistémologique pour penser l'enseignement des grandeurs et mesures et nous discutons l'intérêt, pour les élèves comme pour les enseignants, d'une réflexion didactique sur ces concepts.

Les concepts de grandeur et mesure sont des concepts complexes, en particulier du fait de la polysémie de ces termes, et, malgré leur importance dans l'activité scientifique, leur définition ne fait pas consensus. Dans la partie II, nous discuterons les définitions de ces termes dans la littérature avant de préciser celles que nous adopterons dans la suite de l'article; de plus, ces concepts pouvant être abordés avec différentes postures épistémologiques (opérationnalisme et réalisme), nous les présenterons succinctement. Dans la partie III, nous nous intéresserons à la façon dont les grandeurs sont introduites dans l'enseignement en ciblant quelques exemples de grandeurs, à l'école et au collège : nous traiterons de l'introduction des grandeurs physiques à l'école et des grandeurs électriques au collège. Dans cette partie, nous nous intéresserons également la question du traitement de la mesure et des incertitudes de mesure : nous étudierons la place que les programmes accordent à cette question dans l'histoire de l'enseignement et à l'heure actuelle, dans la scolarité obligatoire (école élémentaire et collège) et au lycée. Nous présenterons ensuite une synthèse des recherches en didactique dans ce domaine, qui portent à la fois sur les conceptions des élèves et étudiants sur la mesure et sur des propositions pour l'enseignement. Enfin, nous nous centrerons sur les enseignants en étudiant leurs conceptions des concepts de grandeur et mesure et nous discuterons la question de la formation des enseignants.

\section{Cadrage théorique}

\section{1 Les concepts de grandeur et mesure}

5 Dans sa thèse, Passelaigue (2011) souligne qu'une première difficulté dans la définition de ces deux concepts scientifiques apparaît lorsque l'on cherche à les isoler, citant par exemple Coelho "en métrologie, le problème de la mesure est lié à la définition des grandeurs» (1993, p. 23) ou encore Courtier qui insiste sur le fait "qu'établir une terminologie non contradictoire et non ambiguë en ce domaine est une entreprise redoutable » (p. 
24). Elle montre que les deux concepts de "grandeur» et de «mesure » semblent indissociables, tant dans leur genèse que dans les ouvrages les évoquant. En effet, leurs définitions, lorsqu'elles existent, sont souvent intimement liées, l'un des concepts définissant l'autre. Ainsi, dans le dictionnaire associé à l'Encyclopédie Universalis par exemple, la grandeur est «le caractère de ce qui peut être mesuré » alors que la mesure est l'«évaluation d'une grandeur par comparaison avec une grandeur de même espèce prise comme référence (unité, étalon) ». On retrouve le même type de définitions croisées dans nombre d'ouvrages ou d'encyclopédies scientifiques. On peut considérer que ces définitions sont "refermées sur elles-mêmes », la grandeur n'étant pas définie en dehors de son lien avec la mesure. Cependant, on peut définir ces deux concepts indépendamment l'un de l'autre, c'est l'objet du paragraphe suivant, dans lequel nous soulignons en outre quelques unes des difficultés qui peuvent résulter de ces définitions pour les élèves.

\section{1.1 Le concept de grandeur}

Dans la littérature, les définitions de "grandeur » sont très variées. Passelaigue (2011) a catégorisé ces définitions, issues de dictionnaires, d'encyclopédies ou d'ouvrages scientifiques. Les principales catégories qu'elle identifie sont listées ci-dessous.

- Grandeur comme immensité : C'est le sens qui est proposé en premier lieu dans certains ouvrages. Ainsi dans le Larousse 2009, la grandeur est tout d'abord le « caractère de ce qui est grand, important, considérable ». Cette définition laisse présager des obstacles liés à ce sens commun du terme grandeur. Comment en effet imaginer une grandeur ayant une valeur de l'ordre de l'infiniment petit alors que ce terme porte en lui une idée d'immensité?

- Grandeur en référence à une grandeur de nature particulière : la longueur. Ainsi le Larousse 2009 propose comme seconde définition de grandeur : «Étendue en hauteur, longueur, largeur, taille, dimension ». Cette définition relève de ce que nous appellerons dans la suite « espèce de grandeur ».

- Grandeur en référence à la variabilité : dans d'autres ouvrages on peut lire des définitions de grandeur relatives à la possibilité d'augmentation et de diminution de l'objet observé «on appelle grandeur tout ce qui peut être augmenté ou diminué » (Quillet, 1961, p. 155). Notons qu'une définition aussi large de la grandeur ne permet pas de définir clairement le concept. Ainsi, comme le soulignent les auteurs de la revue de l'Association des Professeurs de Mathématiques de l'Enseignement Public, « si on appelle grandeur tout caractère d'un objet [...] susceptible de variations chez cet objet, ou d'un objet à un autre, les exemples de grandeurs sont nombreux: la gentillesse, l'agressivité, l'intelligence [...] pour aucune de ces grandeurs on ne saurait parler d'égalité » ( $\left.{ }^{\circ} 46, \mathrm{p} 24\right)$.

- Grandeur en référence à la mesure et au mesurage : comme nous l'avons souligné précédemment, les termes « grandeur » et « mesure » sont très souvent associés, avec des définitions « circulaires » : la grandeur est ce qui peut être mesuré et la mesure consiste à évaluer une grandeur par comparaison avec une grandeur de même espèce prise comme référence.

7 Passelaigue (2011) souligne aussi que le terme grandeur fait partie des « bad words » cités par le physicien Bell dans le magazine «Physics World» d'août 1990 pour qualifier les mots qui sont sur-employés par les physiciens mais qui n'ont pas de définition claire et stable.

Dans la suite de notre étude, nous retiendrons la définition du Vocabulaire International des termes fondamentaux et généraux en Métrologie (VIM, 2008, p VI), qui définit les grandeurs comme "propriétés d'un phénomène, d'un corps ou d'une substance que l'on peut 
exprimer quantitativement sous la forme d'un nombre et d'une référence »'. On parlera alors de grandeur au sens 1. Notons qu'une définition proche de celle-ci n'est apparue en France que tardivement, vers 1650, alors même que les grandeurs ont été le support de la construction des nombres depuis l'antiquité.

9 Le VIM distingue les grandeurs de base (comme la longueur, la masse), et les grandeurs dérivées qui sont des fonctions des grandeurs de base (la vitesse, la masse volumique par exemple), une grandeur de base appartenant à un système de grandeurs tel qu' « aucune grandeur ne puisse être exprimée en fonction d'une autre » (VIM, 2008, p 4). Dans cet article, nous nous intéressons principalement à la construction et à la mesure des grandeurs de base, en particulier lorsque nous étudions les processus de mesurage. La question de la construction et du sens de la mesure des grandeurs dérivées ne sera donc pas abordée ici. Notons que cette question est peu travaillée dans la littérature et nécessiterait des recherches spécifiques.

10 Pour définir les grandeurs, les scientifiques ont comparé les objets selon un certain point de vue, directement ou à l'aide d'un appareil, et construit à partir de ceux-ci des classes d'équivalence. Voyons par exemple comment est définie la grandeur «longueur». Lorsqu'on dispose de deux objets allongés, on peut les porter l'un sur l'autre et tenter de faire coïncider leurs extrémités. Si on y parvient, ces deux objets ont en commun une propriété : on déclare qu'ils ont la même longueur. Tous les objets allongés ayant en commun cette propriété sont regroupés au sein d'une même classe d'équivalence. Si on ne parvient pas à faire coïncider les extrémités de deux objets allongés, l'un est déclaré plus long que l'autre. Si les deux objets ne peuvent être transportés, le passage par un troisième objet peut permettre d'effectuer cette comparaison. Les diverses classes d'équivalence peuvent ensuite être rangées. On définit donc tout d'abord des relations d'équivalence entre objets puis des relations d'ordre entre classes d'équivalence. Lorsqu'on mathématise l'idée de grandeur, on identifie donc la grandeur à une propriété non pas de l'objet mais d'un ensemble d'objets. La notion de grandeur comme propriété commune à un ensemble d'objets équivalents se cache dans la pratique derrière la règle suivante : «dans toute comparaison d'objets on peut, sans altérer le résultat de la comparaison, remplacer chacun des deux objets par un objet équivalent ».

11 Nous appelons « espèce de grandeur ", l'aspect commun à des grandeurs mutuellement comparables. De ce fait, nous considérons comme espèce de grandeur l'ensemble des classes d'équivalence données par l'étude des objets selon un certain point de vue. Une espèce de grandeur, la longueur par exemple, peut selon la situation porter des noms différents: la circonférence, la hauteur, la profondeur... Nous parlons alors de "grandeurs de même nature », puisque ces termes se référent en fait à une seule espèce de grandeur.

12 Nous avons donc défini " grandeur » comme propriété, attribut, qui permet de décrire les objets ou les phénomènes. Cette première description des phénomènes peut conduire à comparer les objets selon un point de vue, la propriété choisie. Ainsi, il est possible de comparer directement deux objets allongés et de déterminer s'ils sont équivalents ou non du point de vue de la longueur, c'est-à-dire d'une espèce de grandeur. Nous pouvons aussi comparer ces deux objets en les plaçant sur les plateaux d'une balance et déterminer s'ils sont équivalents ou non selon un autre point de vue, une autre espèce de grandeur : la masse (au repos). Ces comparaisons directes, instrumentées ou non, sont réalisables à l'aide de procédés divers sans qu'il soit nécessaire de choisir une unité et d'introduire un nombre et donnent toujours le même résultat en un lieu donné. Cette première approche 
qualitative des grandeurs est insuffisante pour des raisons évidentes. En particulier il existe des grandeurs qui ne sont pas comparables directement (l'aire, la force...). Par ailleurs des analogies entre, d'un côté, certaines actions réalisables sur les objets (et sur certaines grandeurs associées), comme la réunion ou le fractionnement, et de l'autre, les opérations sur les nombres ont aussi été recherchées, théorisées. C'est alors que les mathématiques et la mesure entrent en jeu: «L'expérience prend la forme de la mesure, par des instruments, pour connaitre la valeur d'une grandeur (ou de grandeurs) mathématisée(s)» (Paty, 1994, p. 161).

\section{1.2 Le concept de mesure}

\section{Mesure et mesurage}

On définit couramment la mesure par l'«évaluation d'une grandeur par comparaison avec une grandeur de même espèce prise comme référence (unité, étalon)» (Malifaud, 2008) ou encore comme « le rapport de cette grandeur à une autre grandeur de même espèce choisie comme unité » (De Broglie, 1955). Des obstacles linguistiques apparaissent ici encore car l'emploi du terme mesure est source d'ambiguïté : ainsi sous le terme «mesure " peuvent se cacher d'une part l'opération proprement dite de " mesurage » et d'autre part le résultat de cette opération, c'est-à-dire le nombre obtenu grâce au mesurage, et ces écarts d'acception peuvent être sources de confusions. Nous distinguons les termes de mesure et mesurage dans la suite de cet article, même si en métrologie on ne les distingue pas puisque la définition suivante est commune à ces deux termes : « ensemble d'opérations ayant pour but de déterminer une valeur d'une grandeur " (BIPM, 2008). Notons qu'en anglais on trouve aussi une distinction entre "measurement" et "measure", même si le terme de « measurement » peut lui aussi être source d'ambiguïté puisque, comme le terme français " mesure ", il peut être employé pour décrire à la fois l'acte de mesurer et le résultat de la mesure.

\section{Étalon et instrument}

La méthode de mesure la plus simple est la comparaison directe avec un étalon de mesure. Cela suppose le choix de cet étalon de mesure, grandeur de même espèce prise comme référence. Le lien avec le nombre permet alors d'établir des relations mathématiques dans la comparaison de deux objets possédant en commun une même grandeur (l'un étant objet de la mesure, l'autre étant l'étalon choisi). Par exemple, pour reprendre le cas de la longueur, si on peut aligner 3 fois l'étalon de longueur choisi sur un objet allongé, on dira que la mesure de la longueur de cet objet est égale à 3. Ce nombre sera par ailleurs accompagné de son unité. Cependant le mesurage de certaines grandeurs ne peut se faire par comparaison directe. Il nécessite de s'appuyer sur des lois de la physique dont on suppose qu'elles sont invariantes dans les domaines considérés, ce qui nécessite alors une chaîne de mesurage (c'est le cas par exemple pour la mesure des intensités électriques dans les ampèremètres électromagnétiques qui est liée à la loi de Laplace).

15 La mise en place d'un étalon commun est le résultat d'accords scientifiques et politiques ayant nécessité du temps et peut encore aujourd'hui être l'objet de modifications. Une fois choisi, pour satisfaire aux conditions de communication, il doit pouvoir être transmis, dupliqué, en respectant au mieux la référence qu'il représente. Ces «copies » constituent les instruments de mesure les plus simples. Cela implique une chaîne 
d'étalonnage afin de produire des instruments de mesure utilisables par tout un chacun. Puisque l'appareil de mesure est le résultat d'une chaîne d'étalonnage, les appareils de mesure ont des qualités définies par la norme X 07-001 en France, entre autres :

- la sensibilité (aptitude à donner des indications distinctes pour deux valeurs voisines de la grandeur à mesurer) ;

- la justesse (aptitude à donner des indications non entachées d'erreur systématique) ;

- la fidélité (aptitude à donner la même indication pour une même valeur de la grandeur mesurée);

- la finesse (quantification de la perturbation du phénomène par l'appareil de mesure).

Un appareil est dit précis s'il est fidèle et juste. Ces qualités amènent les utilisateurs à préciser les conditions d'utilisation de l'appareil considéré.

\section{Mesure d'une grandeur}

La grandeur que l'on veut mesurer est appelée en métrologie « mesurande » (VIM, 2008). Lorsqu'on mesure une grandeur, sa "mesure » est un nombre déterminé en fonction d'un étalon pris comme référence, qui permet d'élaborer une classe d'équivalence que nous appelons « valeur » d'une grandeur. La valeur d'une grandeur ne dépend pas de sa mesure mais elle peut être exprimée par cette mesure. Par exemple la longueur d'une tige peut être exprimée en centimètres ou en décimètres: les nombres sont différents mais la valeur de la longueur de la tige est indépendante de ces nombres. Cette valeur constitue la classe d'équivalence. Dans le langage courant, on ne fait généralement pas la distinction entre la grandeur et la valeur de cette grandeur. On parle de la "grandeur " d'un objet, par exemple on dit souvent la longueur d'un objet est de $3 \mathrm{~cm}$, il s'agit en fait de la valeur de la longueur, nous parlerons dans ce cas là de grandeur au sens 2 . Il en résulte que pour écrire le résultat de la mesure d'une grandeur, la longueur par exemple, il faut spécifier l'unité choisie : la mesure en centimètres de cette longueur est égale à 3 . Dans l'absolu, toutes les autres formulations génèrent des confusions, soit entre la mesure et la grandeur support de la mesure (cette longueur mesure $3 \mathrm{~cm}$ ), soit entre la grandeur et sa valeur (cette longueur est égale à $3 \mathrm{~cm}$ ); elles sont cependant largement utilisées dans le langage courant pour ne pas alourdir les formulations. De nombreux débats ont eu lieu et sont toujours d'actualité dans la communauté des didacticiens des mathématiques sur la façon dont on doit écrire les calculs sur les grandeurs (voir par exemple Chevallard et al., 2001) : doit-on indiquer les unités à chaque étape du calcul, uniquement à la fin ou encore dans une phrase à la suite des étapes du calcul ? Tout cela laisse présager des difficultés pour les élèves de l'école : que dire de la longueur d'une ligne courbe lorsque les instruments, l'étalon dont on dispose est rectiligne? Comment mesurer des grandeurs continues lorsque les nombres dont on dispose sont des entiers? Quel lien entre étude des grandeurs et construction des nombres? Cette dernière question a été étudiée en didactique des mathématiques par Chambris (2008) qui a mis en évidence des évolutions importantes dans l'enseignement au cours du XXe siècle.

\section{Grandeur « mesurable ", grandeur « repérable ", grandeur « comparable»?}

Nous avons considéré comme grandeur une propriété qui peut être quantifiée, or Le Chatelier souligne que "Tous les phénomènes dont la grandeur est variable ne sont pas nécessairement mesurables» (1914, p. 139). On distingue en physique les grandeurs mesurables et les grandeurs repérables. Le Chatelier qualifie les grandeurs mesurables 
comme étant les grandeurs qui obéissent à deux exigences : la loi d'équivalence et la loi d'additivité. La loi d'équivalence sert à identifier la grandeur proprement dite. La loi d'additivité quant à elle sert à définir la mesurabilité d'une grandeur: "pour qu'une grandeur soit réellement mesurable, il faut encore qu'elle obéisse à la loi d'additivité, c'est-à-dire qu'en réunissant plusieurs corps identiques, sièges de phénomènes semblables, on puisse constituer un système équivalent aux phénomènes isolés dont on peut mesurer la grandeur. Le nombre de ces corps identiques, pris comme terme de comparaison et appelé unité, donne précisément cette mesure» (Le Chatelier, 1914, p. 140). On peut donc dire que lorsqu'on peut définir la somme de deux grandeurs, celles-ci sont dites mesurables, c'est le cas par exemple pour la longueur : si on aligne deux baguettes on obtient une baguette dont la longueur est la somme de la longueur des deux baguettes. En revanche, ce n'est pas le cas pour la dureté, pour laquelle les mesures effectuées ne peuvent être ajoutées. C'est cette absence d'additivité qui différencie les grandeurs repérables des grandeurs mesurables. On retrouve une définition de ce type dans l'encyclopédie Universalis de nos jours : pour Malifaud "on peut repérer des valeurs d'une grandeur et les ordonner selon une échelle numérique par rapport à une valeur stable prise comme point de repère. [...] La mesure demande qu'on sache définir la somme de deux grandeurs de même espèce ou de deux valeurs d'une même grandeur. Cette espèce de grandeur est dite additive. On choisit une certaine valeur comme unité et l'on mesure la grandeur par le rapport entre la valeur observée et la valeur unitaire » (Malifaud, 2008). Nadi (2000) propose quant à lui de distinguer les grandeurs mesurables et repérables en précisant les opérations auxquelles de telles grandeurs se soumettent. Pour les grandeurs mesurables il est possible d'établir une relation d'équivalence, une relation d'ordre, l'addition, et la multiplication par un scalaire. On retrouve ici les deux lois proposées par Le Chatelier pour l'identification de la mesurabilité d'une grandeur. En ce qui concerne les grandeurs repérables (la dureté, l'intensité des tremblements de terre...), Nadi précise que celles-ci sont identifiables par le fait que l'addition et la multiplication par un scalaire ne peuvent être établies pour ce type de grandeurs. La comparaison et la mesure de ces grandeurs repérables imposent d'établir des échelles arbitraires, définies conventionnellement, telles l'échelle de Richter pour les séismes, ou encore l'échelle de dureté.

19 De Broglie (1955) porte un avis différent sur cette distinction entre grandeurs mesurables et repérables. Pour lui, la définition de la mesure d'une grandeur à travers la mesurabilité de celle-ci et la reconnaissance de la grandeur mesurable à l'additivité est trop restrictive. De Broglie considère que la mesure d'une grandeur est un nombre « qui fixe, de façon exacte et indiscutable, son intensité ou seulement son état, à l'égard de quiconque, et qui fournisse ainsi, par simple rapprochement de nombres, le résultat de sa comparaison avec une grandeur quelconque de la même espèce, mesurée de même, en tout autre temps comme en tous autres lieux et circonstances " (De Broglie, 1955, p. 2_08_1). Pour lui, le repérage est aussi une mesure, avec les qualités qu'il lui attribue. Notons que cet extrait met en avant une position philosophique particulière: il existe une valeur «indiscutable» d'une mesure, nous y reviendrons plus loin. Dans la section $\mathrm{C}$ de son encyclopédie de physique, une précision est donnée pour exprimer ce que sont les grandeurs repérables : " elles sont repérables si on sait les classer entre elles par la définition des relations $=,\langle$,$\rangle , ce qui permet d'attacher$ univoquement un nombre à chacune » (De Broglie, 1955, p. 2_12_1).

Une autre question est à prendre en compte lorsque l'on parle des valeurs que peut prendre une grandeur: celle de l'influence ou non de la quantité de matière sur la variabilité de la grandeur choisie. En thermodynamique, on distingue les grandeurs 
intensives (indépendantes de la quantité de matière), des grandeurs extensives qui sont, quant à elles, fonction de la taille du système considéré, ce dernier étant supposé à l'équilibre et homogène.

\section{Fonction et traitement des mesures}

21 Pourquoi mesure-t-on dans la pratique scientifique? Quelles décisions les différents acteurs sont-ils amenés à prendre en fonction de ces mesures?

Dans l'enseignement comme dans la pratique scientifique, la mesure et l'instrumentation ont pour objectif, dans le cadre d'une démarche expérimentale, de définir clairement le phénomène étudié, de construire des faits scientifiques, renouvelables, objets d'une étude scientifique. Les activités faisant appel à la mesure et à l'instrumentation peuvent conduire, entre autres, à tester une hypothèse, à établir une loi (Carnap, 1973), à déterminer des paramètres ou des constantes physiques, à confronter la théorie à l'expérience, à contrôler une théorie, explorer le champ de validité d'une théorie ou d'un modèle, ajuster un modèle. D'un point de vue épistémologique, les mesures servent de test des théories. En effet «les nombres marquent le désaccord par rapport à une théorie, avec une autorité et une finesse qu'aucune technique qualitative ne peut atteindre, et cet écart suffit pour démarrer une recherche» (Kuhn, 1990, p. 280-281). La mesure réalisée dans un cadre expérimental contrôlé peut ainsi permettre de choisir entre deux théories, ce que le débat théorique ne peut faire à lui seul. Avec les mathématiques, les scientifiques sont ainsi passés à des théories, à des modèles validés par leur efficacité à parler du monde. Ces nouvelles théories ont parfois permis d'identifier, de "créer » de nouveaux êtres scientifiques supports de nouveaux modèles, de nouvelles espèces de « grandeur ». Outre l'apparition de nouvelles grandeurs, dans certains cas les théories ont conduit les savants à abandonner des grandeurs qu'ils croyaient avoir bien définies (l'impetus par exemple). Dans d'autres cas, la définition de l'une ou l'autre de ces grandeurs a changé, s'est étoffée (c'est le cas pour la masse par exemple).

23 Quelles que soient les fonctions attribuées au mesurage, les mesures doivent être traitées. Le processus de mesurage comporte en effet deux étapes : le recueil de données (étape qui nécessite de définir la grandeur à mesurer, de sélectionner le matériel de mesure approprié, de définir l'exactitude de la mesure nécessaire et d'organiser le protocole de recueil) et le traitement des données (étape qui définit le protocole auquel les données quantitatives sont soumises). Les valeurs obtenues lors de l'activité de mesurage, accompagnées de leur incertitude, doivent être traitées pour donner des informations sur le phénomène ou l'objet étudié : «le traitement de données est en fin de compte la conversion de données en conclusion sur le monde physique " (Maruani, 1996, p. 1440).

Dans le cadre de la métrologie, deux approches principales de la mesure sont décrites dans le JCGM (Joint Committee for Guides in Metrology) :

- l'approche traditionnelle : l'objectif du mesurage est de déterminer une estimation de la valeur aussi proche que possible de la « valeur vraie », les imperfections dans le mesurage étant appelées « erreurs » (erreur aléatoire qui peut être réduite et erreur systématique qui peut être corrigée). Cette approche peut également être appelée approche " erreur »; l'approche « incertitude » au contraire considère que «l'objectif des mesurages (...) n'est pas de déterminer une "valeur vraie" le mieux possible. On suppose plutôt que l'information obtenue lors d'un mesurage permet seulement d'attribuer au mesurande un intervalle raisonnable de valeurs, en supposant que le mesurage a été effectué correctement " (JCGN-VIM, 2008, p.ix-x). 
Cette approche suggère que «les composantes de l'incertitude de mesure soient groupées en deux catégories, type A et type B, selon qu'elles sont évaluées par des méthodes statistiques ou par d'autres méthodes ".

C'est dans cette deuxième approche que nous nous inscrivons. Comme le dit Perdijon : « il ne suffit donc pas d'un nombre pour exprimer la mesure, il en faut deux : l'estimation la plus probable de la grandeur et l'amplitude de l'intervalle à l'intérieur duquel elle a de grandes chances de se trouver, ce qu'on appelle un intervalle de confiance» (Perdijon, 2012, p. 43). Cette incertitude peut être liée à des erreurs aléatoires (quelle que soit la précision du mesurage, la répétition de la mesure d'une grandeur donnera des résultats qui se répartissent autour d'une valeur moyenne) et à des erreurs systématiques de mesurage. Elles peuvent avoir diverses origines : l'observateur, l'instrument de mesure et l'objet du mesurage. Lippmann considère que l'étalement d'une série de mesures est dû soit à une variation interne (liée au phénomène en jeu donc à la grandeur à mesurer) soit à une variation externe (liée à l'appareil de mesure et à l'expérimentateur). Elle souligne que cette catégorisation est cependant moins simple qu'il n'y parait puisqu'une même variation peut être interne ou externe selon le contexte expérimental (Lippmann, 2005).

\section{2 Deux positions épistémologiques principales}

D'un point de vue philosophique, il existe deux courants de pensée principaux au sujet des grandeurs et mesures : l'opérationnalisme et le réalisme (Perdijon, 1998, 2012).

\section{2. 1 Opérationnalisme}

Pour les opérationnalistes, les grandeurs ne sont pas définies directement mais à travers une succession d'opérations exécutées à l'aide d'instruments : "selon une première école, il ne faut prendre en compte dans un phénomène que les objets qui peuvent être définis par une suite d'opérations, exécutées au moyen d'instruments physiques » (Perdijon, 2012, p. 63).

L'opérationnalisme trouve son origine dans les réflexions d'Ernst Mach et a été formalisé par Bridgman. Dans cette pensée, il est vain de rechercher une quelconque réalité au delà de l'indication donnée par un instrument de mesure. Dans l'école des opérationnalistes, la signification de chaque terme scientifique doit pouvoir être déterminée en spécifiant une opération de vérification bien définie qui lui fournit un critère d'application (Ullmo, 1969, p. 24). Pour Ullmo (1969), les concepts scientifiques sont définis non pas directement, mais à travers un ensemble de procédures déterminées spécifiant les conditions de leur utilisation, procédures qui doivent pouvoir être répétées. Le choix des techniques de mesure joue donc un rôle prépondérant sur la définition des objets scientifiques et sur l'identification des grandeurs pertinentes, puisque les procédures de mesurage sont bien des successions d'opérations répétables, permettant de définir les concepts scientifiques. Dans la pensée opérationnaliste, «[...] il n'y a pas d'une part, l'esprit, de l'autre, les choses, mais détermination réciproque des choses par l'esprit et de l'esprit par les choses [...] il n'y a pas, d'une part, les instruments de mesure, de l'autre, les phénomènes mesurés, mais détermination réciproque du mesurant et du mesuré » (Ullmo, 1969, p. 43). De ce fait, pour Ullmo (1969, p. 25), "C'est la mesure même qui définit la grandeur à mesurer; celle-ci ne préexiste pas à sa mesure, comme une intuition sommaire l'a longtemps fait croire ». Pour lui, les grandeurs sont issues des procédures de mesurages et peuvent varier au fil de l'évolution de la science (Ullmo, 1969). 
30 En outre, la théorie sous jacente a une part dans la construction des êtres scientifiques : « L'expérience n'est pas passivité, enregistrement d'un donné. Elle est activité, sollicitation, construction. L'expérimentation, et même la simple observation, sont des théories en acte » (Ullmo, 1969, p. 97). Concevoir une expérience, un instrument de mesure suppose de s'appuyer sur une théorie. On retrouve ici l'idée que «Toute observation, quel que soit son degré de sophistication, qu'elle soit passive ou accompagnée d'une expérimentation, se fait dans un certain cadre conceptuel » (Perdijon, 2012, p 63). Pour conclure sur ce bref aperçu, dans la pensée opérationnaliste, les grandeurs seraient des objets créés par le scientifique en fonction de la théorie qu'il soutient et qu'il construit, au travers d'instruments permettant de mettre en évidence des relations répétables.

\section{2. 2 Réalisme}

31 Le second courant philosophique dans lequel peut se placer un scientifique pour parler des grandeurs et de la mesure est le réalisme. Cette position philosophique s'appuie sur l'idée de l'existence d'un réel en dehors de la mesure. Selon le point de vue réaliste, le but de la science est de décrire ce à quoi ressemble réellement le monde et d'en trouver une explication. L'hypothèse de départ du réalisme est que le monde réel existe en dehors de notre pensée et qu'il possède une organisation interne, dont les manifestations extérieures sont des phénomènes observables par l'homme. Le terme de réalisme est aujourd'hui associé à des courants de pensée divers et à ce terme est maintenant accolé un adjectif précisant ce courant de pensée: on parle par exemple de réalisme naïf, rationnel, opératoire, ou encore structural.

Le réalisme naîf postule une vérité connaissable et absolue, indépendante des observateurs comme des instruments de mesure. Dans cette perspective, les énoncés de la théorie et les phénomènes du monde réel sont isomorphes. Notre connaissance du monde est encore imparfaite du fait des limitations de nos instruments, mais nous progressons vers la vérité. Une théorie qui donne une description correcte du monde réel a caractère de vérité.

33 Le scientifique d'aujourd'hui ne peut plus se définir réaliste au sens originel du terme. Les révolutions scientifiques ont montré que la position réaliste que d'Espagnat (1994) appelle « conventionnelle », n'est pas tenable : « Le réalisme scientifique naïf, qui impliquerait une correspondance quasi absolue entre nos théories et la réalité n'est pas raisonnable à l'heure de la physique quantique et de la relativité» (Guay, 2007). Le fait que dans toute expérimentation interviennent différentes théories (pour décrire le système et pour concevoir les instruments) a été pointé par différents auteurs réalistes (Hacking, 1983, par exemple).

34 Le réalisme d'aujourd'hui prend en compte cette évolution historique des connaissances scientifiques et on parle maintenant de réalisme scientifique. Le réalisme scientifique peut être caractérisé par les trois propositions suivantes (Sankey, 2002, Esfeld, 2006) :

- l'existence et la constitution de la nature sont indépendantes des théories scientifiques et ne dépendent pas du fait qu'il y ait ou non des personnes qui construisent des théories scientifiques;

- la constitution de la nature détermine lesquelles de nos théories scientifiques sont vraies. Par conséquent, si une théorie scientifique est vraie, les objets que pose cette théorie existent et leur constitution rend vraie la théorie en question; 
- les sciences sont capables de nous donner un accès cognitif à la constitution de la nature. En particulier, nous avons à notre disposition des méthodes d'évaluation rationnelle applicables à des théories scientifiques concurrentes qui sont capables d'établir, au moins de manière hypothétique, laquelle de ces théories ou interprétations concurrentes est la meilleure au niveau de la connaissance.

de la connaissance que nous en avons " (1987, p. 258). Chalmers parle de réalisme non figuratif «dans la mesure où il ne contient pas une théorie de la correspondance de la vérité avec les faits » (Chalmers, 1987, p. 258). Pour le réaliste non figuratif, il s'agit d'évaluer le degré de réussite des théories à saisir un aspect du monde. Il ne s'agit pas de dire si les théories parviennent à décrire ou non le monde tel qu'il est réellement puisque l'accès au monde n'est pas indépendant des théories que nous élaborons. Dans cette philosophie, il est difficile d'identifier la réalité des grandeurs : existent-elles intrinsèquement ou sont-elles le résultat d'opérations faites sur le monde? On peut ici considérer les grandeurs selon deux points de vue :

- les grandeurs sont intrinsèques et existent indépendamment de leur identification par l'expérimentateur ;

- ce qui sert à décrire le monde appartient à la théorie. La théorie précède l'observation, les expériences et observations sont faites pour tester les théories. Celles-ci nous guident pour savoir quelles observations sont pertinentes ou non par rapport au phénomène étudié. La grandeur est alors un outil de la théorie et n'a donc d'existence qu'au travers de celle-ci. Cependant, les théories s'appliquant dans et hors de l'expérience, on peut considérer que, dans cette approche, les grandeurs, outils théoriques, existent en dehors de l'activité expérimentale.

\section{Le réalisme opératoire}

Le Roy, cité par Ullmo (1969), a proposé une distinction entre le fait brut et le fait scientifique, indispensable pour mettre en évidence qu'un fait brut (le tonnerre, les éclipses, les phases de la Lune...) est porté par une théorie préalable qui peut être rudimentaire et que Bachelard nomme connaissance préscientifique : «Si l'on voulait que les faits bruts fussent présentés à l'observateur indépendamment de toute activité préalable de l'esprit, on pourrait dire, sans glisser au paradoxe, qu'il n'y a pas de fait brut » (Ullmo, 1969, p. 121). Le phénomène scientifique ici est perçu non plus comme étant le fait d'une réalité qui s'impose à l'esprit, mais comme un résultat d'une série d'opérations conduites pour percevoir le monde de façon plus objective.

Dans les sciences d'aujourd'hui, les théories précèdent les instruments, de sorte que l'instrument physique est une théorie réalisée, concrétisée. Dans cette philosophie de la grandeur (réalisme opératoire), il s'agit pour le scientifique de construire un modèle 
pertinent permettant de reconstruire le réel. Nous reconnaissons une qualité donnée à un objet parce que nous avons conçu un modèle nous permettant de la reconnaître. Perdijon (1998) déclare se placer dans une optique réaliste de ce type, dans laquelle il s'agit d'élaborer un modèle permettant de reconstruire le réel à partir du sensible. Il existerait donc « quelque chose » indépendant de la mesure : la grandeur, celle-ci ne s'imposant pas immédiatement à l'esprit, mais étant le résultat d'une succession d'opérations permettant de la définir.

Zahar (2000) défend une forme de réalisme structurel (ou structural). Pour lui, la seule réalité que la science réussit à atteindre et à connaître objectivement est constituée par la «structure ». Le monde est structuré rationnellement et notre esprit peut dégager et connaître ces structures. Des discussions existent encore autour de la définition physique de la structure. La structure serait l'ensemble des relations qui la composent, et, selon Worrall (1989) qui défend lui aussi cette forme de réalisme, la relation est donnée par les formules mathématiques de la physique. Zahar et Worrall s'appuient sur la position de Poincaré selon laquelle les relations sont la seule chose que nous pouvons connaître du monde. Dans cette optique philosophique, les relations qui relient les objets sont la manière dont les objets existent. Les grandeurs sont donc définies au travers des relations qu'elles entretiennent. Le réalisme structural admet donc l'existence d'une grandeur, une propriété concrète, à laquelle il est possible d'appliquer une description en termes structuraux, "c'est-à-dire une description qui les représente comme étant invariantes sous certaines transformations de symétrie" (Esfeld pour Barberousse, 2008, p 14). Dagognet défend lui aussi une position qu'il qualifie de réaliste lorsqu'il parle de mesure. Ainsi il considère que "la mesure permet d'accéder à l'essence de la chose, en même temps qu'à sa relation avec les autres choses" (1993, p. 267) et déclare que : « oui, la mesure est réaliste. Elle est la révélation de la chose. Elle est ce qui l'éclaire et ce qui permet de la comprendre. Oui j'ai développé une théorie réaliste, et je ne vois pas comment faire autrement. C'est très limité mais c'est ainsi.» (1993, p. 269). Cette analyse montre que les réalistes de toute obédience considèrent que la grandeur existe en soi, comme le signale Perdijon.

41 Les deux positions philosophiques opérationnaliste et réaliste se distinguent donc par la croyance ou non en l'existence même des grandeurs indépendamment de toute activité de mesurage: peut-on, doit-on parler de grandeur sans parler de mesure? Pour les opérationnalistes cela n'a pas de sens, la grandeur n'ayant pas d'existence en dehors de la mesure, alors que pour les réalistes les grandeurs existent en soi, indépendamment du processus de mesurage. C'est la question du statut que l'on attribue à la grandeur qui est essentielle ici. Or nous avons vu précédemment que c'est lorsqu'il s'agit de définir le concept de grandeur que l'on rencontre le plus de difficultés et une absence de consensus dans la communauté scientifique. Pour le concept de mesure, même si la définition de la mesurabilité ne fait pas consensus, les définitions de la mesure et du mesurage sont beaucoup plus « homogènes » que pour le concept de grandeur. On peut considérer que les définitions $\mathrm{du}$ concept de grandeur sont influencées par les positions épistémologiques de leurs auteurs. Si on reprend la catégorisation de ces définitions données par Passelaigue (2011), on peut penser que les définitions en référence à la mesure et au mesurage correspondent à une position opérationnaliste, les autres définitions semblant correspondre davantage à une posture réaliste, postulant l'existence intrinsèque de la grandeur indépendamment de sa mesure, comme on peut le voir chez De Broglie par exemple. 


\section{Les grandeurs et mesures dans l'enseignement}

Dans cette partie nous nous intéressons à l'enseignement des grandeurs et de la mesure de l'école jusqu'au lycée, en nous questionnant notamment sur les postures épistémologiques des concepteurs des programmes. Nous nous intéressons tout d'abord à la façon dont les grandeurs sont introduites dans l'enseignement primaire et secondaire à partir de quelques exemples (partie III. 1), avant de considérer plus spécifiquement l'enseignement de la mesure et du mesurage et la notion d'incertitude (partie III. 2).

\section{1 La construction des grandeurs à l'école et au collège}

\section{1. 1 Les grandeurs physiques à l'école élémentaire}

En France, à l'école élémentaire, le thème " grandeurs et mesure » figure explicitement dans les programmes de mathématiques, en cycle 2 (grades 1 et 2) comme en cycle 3 (grades 3 à 5) (MEN, 2008a), mais l'étude des grandeurs commence dès le cycle 1. Dès la maternelle l'enfant doit manipuler des objets de formes et de dimensions variées. L'examen de leurs caractéristiques lui permet rapidement de se doter de catégories simples, d'abord dichotomiques (grand/petit). En multipliant les comparaisons, l'enseignant doit amener les enfants à mieux distinguer divers types de critères (dont la taille, la masse par exemple) et à se livrer à des classements, des rangements suivant ces critères, c'est-à-dire selon des grandeurs. C'est ensuite au cycle 2 qu'on voit apparaitre le concept de mesure dans les programmes. Les situations qui permettent de construire les concepts sont progressives :

- comparaison directe : juxtaposition, superposition, mise en regard de deux objets ;

- comparaison indirecte : recours à un objet intermédiaire, à un instrument de report ou transformation de l'un des objets pour le rendre comparable à l'autre ;

- mesurage, en utilisant un étalon arbitraire puis conventionnel (la grandeur unité) et en associant un nombre à la grandeur (le nombre de « reports » nécessaires de la grandeur unité).

Cette dernière méthode marque l'accès à la mesure au sens mathématique du terme, mais il est spécifié dans les documents d'accompagnement que «Les premières activités visent à construire chez les élèves le sens de la grandeur, indépendamment de la mesure et avant que celleci n'intervienne. Le concept s'acquiert progressivement en résolvant des problèmes de comparaison, posés à partir de situations vécues par les élèves, suivis de moments d'institutionnalisation organisés par le maitre » (MEN, document d'accompagnement des programmes 2002, p. 35), et «Les activités de comparaison directes ou indirectes sont essentielles. C'est à travers elles que l'élève accède aux grandeurs considérées et distingue progressivement la longueur d'un objet de la place qu'il occupe ou sa masse du volume qu'il occupe " (MEN, document d'application des programmes du cycle 2, 2002, p. 29).

Une approche extrêmement voisine est proposée dans les standards NTCM aux Etats-Unis (NCTM, 2000), ou encore en Nouvelle Zélande, où cinq étapes sont clairement identifiées dans le processus de mesurage: «identifying the attribute, comparing and ordering, nonstandard units, standard units, application " (ME, 2009). Dans ces différents textes officiels, on peut noter l'importance accordée à la construction de chaque grandeur par des activités de comparaison avant et en dehors de toute activité de mesurage. 

l'école primaire. D'un certain point de vue, les grandeurs sont construites à partir de comparaisons directes puis indirectes, donc d'activités manipulatoires qui peuvent donner aux grandeurs une signification opératoire, ce qui se rapprocherait d'une position opérationnaliste. Cependant les concepteurs de ces programmes préconisent de travailler sur les grandeurs avant et en dehors de toute activité de mesurage, ce qui peut laisser penser qu'ils postulent l'existence des grandeurs indépendamment de l'observateur, ce qui peut aussi laisser penser que leur posture est plus proche du réalisme, mais cette posture n'est en aucun cas explicitée.

Notons que cette approche des grandeurs est relativement récente, en France tout au moins, puisqu'une étude des manuels français de sciences et de mathématiques de 1945 à nos jours a montré qu'avant les années 70, les grandeurs étaient introduites à l'école élémentaire comme ce que l'on mesure avec l'instrument ad hoc (Favrat et Munier, 2007). Aborder les grandeurs en dehors du nombre, à partir d'activités de comparaison directe, indirecte, avant l'introduction de l'étalon arbitraire est ainsi une proposition qui a suivi le mouvement de la réforme des mathématiques modernes et des activités d'éveil en sciences.

Passelaigue (2011) s'est interrogée sur les raisons de ce basculement dans la façon d'introduire les grandeurs à l'école. Elle montre que le mouvement des mathématiques modernes, conduit par des mathématiciens et des enseignants de mathématiques, a été nourri par les travaux de psychologues, Piaget notamment. En effet dans leurs travaux sur la construction des grandeurs, Piaget et Inhelder (1968) ont montré que certains enfants pourtant capables de mesurer n'étaient pas "conservants", montrant par là même qu'une centration sur la mesure pouvait masquer un défaut de conceptualisation des grandeurs. Ces auteurs ont souligné que l'entrée dans la mesure n'est pas forcément le moyen le plus efficace pour construire les connaissances liées à la grandeur. La pensée constructiviste, soutenue par Piaget, qui insiste sur la part active que doivent prendre les élèves dans la construction des connaissances et le contexte de la réforme ont favorisé l'émergence de nombreuses propositions dans le sens d'un travail sur la grandeur préalable à l'introduction de la mesure, en France comme dans de nombreuxpays (voir par exemple Diénes et al., 1970, pour le Canada).

considérer que l'on a basculé dans les années 1970 d'une posture opérationnaliste à une posture réaliste ? On ne peut en aucun cas l'affirmer puisque rien n'est explicite dans les différents textes sur le statut « épistémologique » des grandeurs. La question de l'existence ou pas des grandeurs indépendamment de la mesure est en fait davantage une question de physicien, ce qui peut expliquer que les mathématiciens qui ont porté la réforme des mathématiques modernes ne s'en soient pas emparés.

Passelaigue (2011) s'est intéressée aux pratiques déclarées des enseignants pour l'introduction des grandeurs et de la mesure à l'école. Elle montre que pour les enseignants, l'objectif des activités de mesurage est le plus souvent l'introduction de l'unité conventionnelle avec les outils adéquats, ou encore l'introduction de nouvelles unités de mesure, multiples et sous multiples de l'unité conventionnelle, mais pas l'occasion d'un travail sur le sens de la mesure ou de l'unité. Elle montre aussi qu'un certain nombre d'enseignants mettent peu, voire pas en œuvre d'activités de comparaison avant la mesure. Lorsqu'ils le font, respectant en cela la progression indiquée par les textes, ils le font souvent uniquement pour la longueur, grandeur pour laquelle les propositions d'activités sont nombreuses dans les documents d'application et 
d'accompagnement. La démarche n'est pas transférée aux autres grandeurs abordées à l'école. Certains enseignants semblent ne pas être convaincus de l'intérêt de telles pratiques et, lorsqu'on évoque les comparaisons, ils pensent souvent comparaison de nombres (résultats de mesurage) et non comparaison directe de grandeurs.

La question des enjeux didactiques des approches préconisées dans les programmes est donc une question importante. En France comme à l'étranger, de nombreuses études se sont penchées, en didactique de la physique et en didactique des mathématiques, sur l'enseignement des grandeurs. De nombreux auteurs soulignent l'importance de conceptualiser chaque grandeur avant de la mesurer. Par exemple, Pressiat (2009) considère qu'il est « essentiel de comprendre de quelle grandeur on parle avant de s'intéresser à la question de sa mesure ». Rouche (2006, p. 103) adopte le même point de vue: "il est nécessaire de savoir d'abord ce que sont les grandeurs avant d'apprendre à les mesurer ». Macdonald (2011) souligne que, si de nombreuses séquences pour l'apprentissage de la mesure sont présentées dans la littérature, la plupart sont similaires dans leurs progressions: de l'identification de la grandeur et de l'utilisation d'unités arbitraires jusqu'a l'utilisation des unités conventionnelles (parmi ces études on peut citer Brousseau, 1991-1992, Douady et al., 1989, Perrin Glorian, 2002, Clements and Stephan, 2004, Kospentaris et al, 2011, Boulton-Lewis et al., 1996). Il y a donc consensus chez de nombreux chercheurs en didactique sur la façon d'introduire les grandeurs.

Dans une revue de la littérature anglo-saxonne Macdonald (2011) identifie plusieurs étapes dans la compréhension de la mesure chez les jeunes élèves :

- Aux alentours de 5 ans les élèves commencent à apprendre à utiliser les mots qui représentent une « quantité » de certaines grandeurs (Clements and Stephan, 2004);

- L'étape suivante est de comparer deux objets directement et de reconnaitre l'égalité ou l'inégalité (Boulton-Lewis et al., 1996) ;

- Les élèves sont alors prêts à apprendre à mesurer en associant un nombre à la grandeur (Clements and Stephan, 2004).

Elle souligne que de nombreux chercheurs tendent à s'accorder sur le fait que ces idées forment les fondements de la compréhension de la mesure. Elle précise toutefois que des débats persistent entre les chercheurs sur l'ordre de ces étapes. En effet, certains auteurs considèrent que les activités de comparaison et de mesure ne doivent pas être successives mais simultanées, la mesure participant ainsi à la construction du sens de la grandeur. Par exemple, Hiebert (1981) considère que "It would be more productive to involve children in a variety of concrete measuring activities than to wait until they develop these reasoning concepts. For most students, understanding of conservation and transitivity will develop alongside their understanding of measurement ". D'autres chercheurs accordent aussi un rôle plus important à la mesure dans la conceptualisation de nouvelles grandeurs. Par exemple, Mantyla et Koponen (2006) ont cherché à comprendre le rôle de la mesure dans la création des grandeurs physiques et ils ont étudié le cas de la température. Ces auteurs considèrent que la construction du sens des grandeurs implique nécessairement un ensemble d'expériences et de mesures.

Dans sa thèse, Passelaigue (2011) a explicitement questionné le rôle et l'intérêt de l'approche des grandeurs préconisée dans les instructions officielles. Pour le cas de la grandeur masse, elle a montré que les activités de comparaison sont une aide à la conceptualisation: notamment les élèves ayant vécu une situation de comparaison différencient mieux la masse du volume que ceux qui entrent directement dans des activités de mesurage à l'aide d'un instrument du quotidien affichant la référence 
conventionnelle (le gramme). De plus, l'introduction d'un étalon arbitraire joue un rôle important dans la construction du sens de la mesure: "L'unité de mesure d'une grandeur n'est pas une donnée immédiate, absolue, mais est créée mentalement par celui qui mesure». Passelaigue montre que le concept d'unité de mesure et son aspect arbitraire et conventionnel sont construits dans ces activités, ce qui n'est pas le cas lors d'une entrée par l'instrument de mesure des masses avec une unité conventionnelle imposée d'emblée. Elle montre aussi que dans le travail à l'aide d'étalons arbitraires, la construction du sens de la mesure participe à la construction du sens de la grandeur. Elle montre ainsi l'intérêt de l'approche préconisée dans les IO mais nous considérerons que la question du rôle de la mesure dans la conceptualisation des grandeurs reste à approfondir.

\section{1. 2 Les grandeurs électriques au collège}

Si on se penche maintenant sur les programmes d'enseignement du secondaire, on constate que, pour d'autres grandeurs, on ne retrouve plus l'approche développée au primaire. Au collège, nous considérons l'exemple de l'introduction des grandeurs électriques intensité et tension en 4e puis en $3 e$.

En classe de $4 \mathrm{e}$, les élèves rencontrent pour la première fois les grandeurs intensité et tension dans le cas du courant continu. L'intitulé de cette partie du programme est explicite: "Intensité et tension, deux grandeurs électriques issues de la mesure: quelles grandeurs électriques peut-on mesurer dans un circuit?». Les élèves doivent apprendre que l'intensité (respectivement la tension) d'un courant électrique se mesure avec un ampèremètre branché en série (respectivement un voltmètre branché en dérivation) et connaître les unités d'intensité (Ampère) et de tension (Volt). L'approche préconisée diffère fortement de celle utilisée à l'école élémentaire. Les grandeurs sont introduites d'emblée comme ce que mesurent les instruments et on ne voit plus la trace d'activités de comparaison destinées à donner du sens à ces grandeurs électriques avant la mesure. On est ici dans un cas où la mesure nécessite une chaîne de mesurage et où le principe de fonctionnement des instruments n'est pas accessible aux élèves. Pour donner du sens aux grandeurs, les enseignants ont recours à une explication au niveau microscopique et utilisent différentes analogies (analogie hydraulique, mécanique avec des trains, etc.). Certes, le mesurage de ces grandeurs ne peut se faire par comparaison directe avec un étalon comme dans le cas de la longueur ou de la masse, mais on pourrait imaginer, pour l'intensité, des activités de comparaison de l'éclat d'ampoules ou de vitesses de rotation de moteurs, par exemple, pour introduire la grandeur permettant de quantifier ces phénomènes (l'intensité). On peut penser que le choix qui est fait de présenter ces grandeurs comme issues de la mesure est porteur d'une position épistémologique opérationnaliste.

En classe de 3e, les élèves sont confrontés au courant alternatif. Actuellement, le titre de la partie concernant le courant alternatif est «tension continue et tension alternative : qu'estce qui distingue la tension fournie par le secteur de celle fournie par une pile?»(MEN, 2008b). La distinction entre courant continu et alternatif se fait à partir de la forme du signal visualisé sur un oscilloscope. Les valeurs efficaces d'intensité et de tension sont ensuite introduites dans un chapitre intitulé "mesure d'une tension: qu'indique un voltmètre utilisé en "alternatif" ?» et la tension efficace est définie comme ce que mesure le voltmètre. Les élèves doivent savoir que les valeurs des tensions alternatives indiquées sur les alimentations ou sur les récepteurs usuels sont des valeurs efficaces et connaître la relation reliant valeur efficace et valeur maximale. On pointe ici encore dans les 
programmes une définition uniquement opératoire de la tension efficace : c'est ce que mesure le voltmètre.

On peut noter ici l'aspect paradoxal de cette formulation: la valeur efficace est définie comme ce que mesure un instrument, alors que si l'on s'en tient à la définition de la mesurabilité de Nadi par exemple, cette grandeur n'est pas mesurable puisque, hormis dans un circuit uniquement résistif, on ne peut pas additionner les valeurs efficaces.

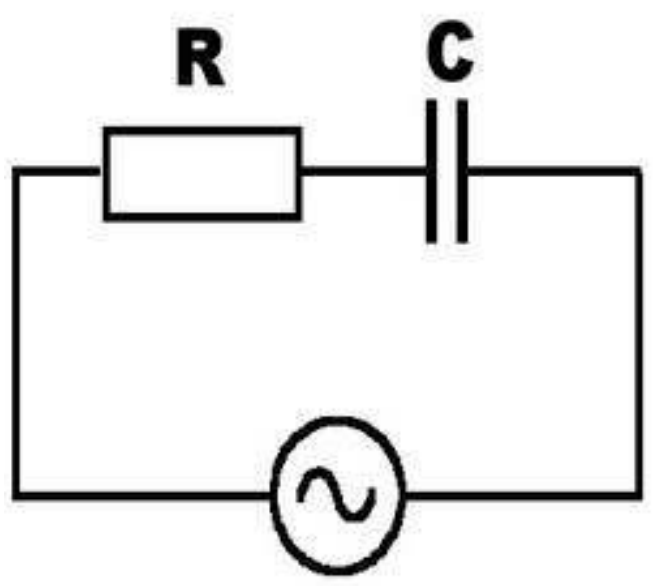

$U \neq U r+U c$

En effet la valeur numérique lue sur le voltmètre traduit un phénomène rapidement variable et la valeur affichée ne correspond donc pas à une mesure mais prend en compte

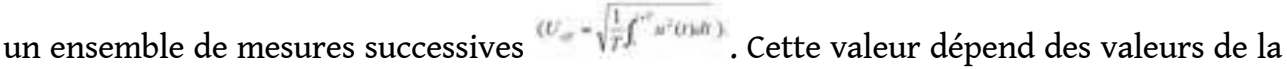
grandeur et de leur répartition dans le temps, c'est-à-dire non seulement de l'amplitude mais aussi de la forme du signal. Or, dans les définitions de la mesurabilité d'une grandeur, aucun des auteurs cités précédemment (partie II.1.1) n'envisage le cas des valeurs efficaces en électricité dont la non additivité des mesures est due au fait que ce sont des valeurs moyennées.

Les programmes quant à eux n'insistent pas sur cette particularité des valeurs efficaces et une des conséquences est que même les étudiants dans les cycles universitaires supérieurs ont encore des difficultés importantes et appliquent abusivement les lois d'additivité des tensions dans des circuits alternatifs (Lascours et Calmettes, 1998, Robert et al., 2008). Robert et al. considèrent qu'une certaine pratique de la mesure et de son utilisation, élaborée avant l'étude de l'électricité, ainsi que les connaissances acquises sur le courant continu, se constituent en obstacle, à la fois épistémologique et didactique, lors de l'introduction de phénomènes rapidement variables dans le temps. En effet, d'un point de vue épistémologique, depuis le début de leur scolarité, les élèves ont été confrontés essentiellement soit à des mesures de grandeurs indépendantes du temps (mesure de la longueur d'un objet par exemple), soit à des mesures instantanées de grandeurs susceptibles d'évoluer dans le temps (mesure de la température lors d'un changement d'état). La seule grandeur moyennée sur le temps rencontrée est la vitesse moyenne d'un mobile. Or, en électricité, en régime variable, il apparaît une nouvelle grandeur : la valeur

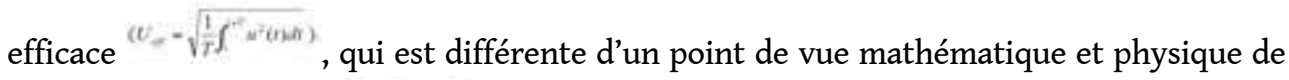

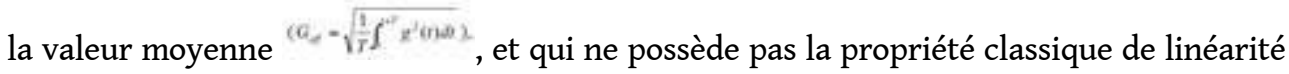
(et donc d'additivité) de cette dernière. Lorsqu'ils abordent l'étude des courants variables, 
pour comprendre la signification des valeurs numériques obtenues, les élèves doivent non seulement savoir ce qu'est une grandeur et une mesure mais aussi ce que représente une valeur moyennée et de quoi elle dépend. Ils doivent donc à la fois prendre conscience qu'ils ne mesurent plus «la même chose » qu'en courant continu, bien qu'ils utilisent parfois les mêmes instruments et, de plus, que les mesures obtenues n'ont plus les mêmes propriétés.

Notons que là encore l'introduction des valeurs efficaces dans les programmes a considérablement évolué au cours du temps. Si actuellement les valeurs efficaces sont définies comme ce que mesure le voltmètre, l'approche utilisée dans les programmes antérieurs était beaucoup plus phénoménologique (Langlois et al., 2011). En effet, lors de l'introduction des valeurs efficaces au début du XXe siècle, les programmes et manuels faisaient au départ référence à la fois aux effets du courant, à la mesure et au calcul de ces grandeurs.

60 Les références aux effets disparaissent ensuite progressivement puisque la différentiation entre les deux types de courants se fait à partir de la visualisation de la tension à l'aide d'un nouvel instrument,(l'oscilloscope) et le calcul mathématique de la valeur quadratique moyenne n'apparaît plus non plus depuis les années 1990 du fait de l'introduction beaucoup plus précoce des valeurs efficaces dans la scolarité, à un âge où les élèves ne disposent pas encore des outils mathématiques suffisants. Peu à peu, cette notion a donc perdu toute référence phénoménologique et il s'en suit une simple définition opératoire de la relation entre valeur efficace (lue sur un multimètre) et valeur maximale (observée sur un oscilloscope). Notons que l'évolution observée ici dans les programmes est inverse de celle observée pour l'école où l'entrée par la mesure a été remplacée par une entrée explicitement centrée sur la grandeur avant les activités de mesurage.

61 On peut penser que les difficultés des étudiants sont dues en partie au fait que, dans leur scolarité, ils sont rarement amenés à se questionner sur le statut des grandeurs ou encore sur le sens de la mesure, sur la différence entre mesurabilité, repérabilité, etc. Ils doivent construire, manipuler, de nombreuses grandeurs, mais il manque un travail explicite sur ce qu'est une grandeur, sur la manière dont les grandeurs sont construites, voire sur les différentes positions épistémologiques possibles concernant les grandeurs. On peut penser aussi que l'introduction d'éléments de métrologie pourrait aider les élèves à construire le sens de la mesure. Certes, des activités telles que celles préconisées à l'école, avec l'utilisation des étalons arbitraires en particulier, ont montré leur efficacité, mais nous avons vu qu'elles sont insuffisamment pratiquées par les enseignants (Passelaigue, 2011). D'autres recherches ont montré que des séquences de classe dans lesquelles les élèves sont amenés à concevoir, voire à fabriquer, des instruments de mesure favorise la construction à la fois du sens de la grandeur et du sens de la mesure (Merle et Munier, 2003, Munier et Merle, 2012), mais de telles activités sont relativement marginales et l'instrumentation, au sens métrologique du terme, est peu exploitée dans l'enseignement primaire et secondaire. Au collège notamment, les élèves utilisent de nouveaux instruments de mesure mais leur fonctionnement ne fait pas l'objet d'une exploitation et l'utilisation de ces instruments a souvent pour objectif essentiel une formalisation mathématique, notamment par l'établissement de relations entre les grandeurs. Séré souligne que c'est l'aspect de plus en plus technologique de l'instrumentation qui réduit la possibilité de l'apprentissage du savoir-mesurer. Cet apprentissage est rendu difficile au collège et au lycée du fait de la complexité des appareils, ce qui peut conduire à des 
confusions pour les étudiants (que mesure-t-on avec un voltmètre, un multimètre, un oscilloscope, quel sens ont ces mesures, quel usage peut-on en faire ?). De ce fait, nous considérons qu'il est d'autant plus judicieux de l'entreprendre avec de jeunes élèves pour lesquels l'instrumentation reste modeste et « transparente ».

\section{2 Le traitement des résultats de mesure et la notion d'incertitude}

62 Si l'on veut que l'enseignement scientifique et technique ne soit pas déconnecté des activités professionnelles liées à la mesure et à l'instrumentation (pratiques des chercheurs ou pratiques des techniciens liées à des activités de mesurage, de contrôle et d'entretien), il convient de former les élèves à la mesure, aux activités de mesurage, aux traitements des données issues de ces activités et par là même à l'instrumentation. Or, actuellement en France, il n'y a pas d'enseignement spécifique sur la mesure, c'est-à-dire d'enseignement de métrologie (Cotteret, 2003), et ce même au niveau universitaire. Cette absence d'enseignement dédié uniquement à la mesure ne signifie pas pour autant qu'elle n'est pas prise en compte dans la scolarité. Ainsi la mesure apparait dans les programmes dès l'école élémentaire et les nouveaux programmes de lycée (MEN, 2010) lui donnent même une place croissante. Nous présentons dans cette partie tout d'abord un historique de l'enseignement de la mesure en France depuis le début du XXe siècle avant de faire le point sur les programmes actuels, de l'école au lycée, puis nous ferons une synthèse de la littérature didactique dans ce domaine.

\section{2. 1 Historique de l'enseignement de la mesure}

63 En sciences physiques, on a assisté au cours des cent dernières années dans l'enseignement français à une évolution des pratiques, des enjeux mêmes du mesurage et de l'importance qu'on lui donne par rapport à l'observation lors de l'expérimentation. Cette évolution de la place de la mesure dans l'enseignement des sciences physiques au lycée depuis 1902 a été retracée par Séré $(2007,2008)$. Elle montre que durant la première moitié du vingtième siècle, le mesurage a été enseigné en tant que savoir-faire, les mesures étant peu (ou pas) utilisées, mais qu'une centration s'est faite ensuite progressivement sur le traitement des données et sur l'évaluation de la qualité de la mesure, en lien avec les théories statistiques. Cette évolution des pratiques et des enjeux du mesurage est liée également aux fonctions, aux buts attribués aux activités expérimentales, qui ont considérablement évolué avec le passage d'un enseignement basé sur la monstration et l'induction à un enseignement basé sur une démarche d'investigation. Séré distingue trois grandes étapes dans l'histoire de l'enseignement du mesurage : passage de la « valeur vraie » et de l'erreur (années 1907-1945) à l'incertitude (jusqu'en 1968) puis à la mesure considérée comme variable aléatoire, ce dernier passage constituant un véritable tournant dans l'histoire de l'enseignement du mesurage. Elle montre que l'introduction de ce raisonnement statistique modifie profondément le traitement des mesures. Les incertitudes sont estimées et le « dogme des encadrements est abandonné » au profit de "l'intervalle de confiance » associé à un taux de confiance. Cette évolution de l'enseignement de la mesure, et particulièrement la problématique des erreurs et des incertitudes, a suivi l'évolution de la métrologie, même si l'aspect probabiliste met du temps pour être introduit officiellement dans l'enseignement français. 


\section{2. 2 Les programmes actuels de l'école au lycée}

\section{Dans la scolarité obligatoire (école élémentaire et collège)}

l'issue de la scolarité obligatoire les élèves doivent être capables « d'effectuer des mesures à l'aide d'instruments, en prenant en compte l'incertitude liée au mesurage » (MEN, 2006). Pour cela, ils doivent maîtriser les principales unités de mesure, savoir les associer aux grandeurs correspondantes, comprendre qu'à une mesure est associée une incertitude ainsi que la nature et la validité d'un résultat statistique (MEN, 2008b). Les instructions précisent que les enseignants de mathématiques et de physique doivent s'associer pour développer chez les élèves un "Mode de pensée statistique dans le regard scientifique sur le monde ": "L'observation [...] peut être complétée par l'utilisation d'appareils de mesure et par l'exploitation mathématique des résultats qu'ils fournissent. L'exploitation de séries de mesures, la réflexion sur leur moyenne et leur dispersion [...] introduisent l'idée de précision de la mesure et conduisent à une première vision statistique du monde ». Ils doivent en particulier confronter les élèves au problème de la variabilité de la mesure: "De nombreuses activités doivent intégrer la notion d'incertitude dans l'acte de mesurer et développer l'analyse des séries de mesures. [...] Plusieurs mesures indépendantes d'une même grandeur permettent ainsi la mise en évidence de la dispersion naturelle des mesures. » (thème de convergence 1, MEN, 2008b).

Concernant le traitement des données, dès l'école primaire les élèves doivent «apprendre progressivement à trier des données, à les classer, à lire ou à produire des tableaux, des graphiques et à les analyser. " (MEN, 2008a). En fin de scolarité obligatoire, ils doivent être capables d'" utiliser et construire des tableaux, des diagrammes, des graphiques et savoir passer d'un mode d'expression à un autre " (MEN, 2006). Au collège, les élèves abordent "les notions fondamentales de statistique descriptive (maximum, minimum, fréquence, moyenne) " et les notions de chance ou de probabilité (MEN, 2006). Il est souligné que le travail sur les données issues de mesurage est un lieu privilégié pour montrer les convergences entre les disciplines scientifiques: "La construction et l'utilisation d'un tableau ou d'un graphique à partir d'une série de données [...] sont d'autres occasions de nouer des liens avec les mathématiques. "(MEN, 2008b). En fin de scolarité obligatoire, les élèves doivent donc maîtriser des compétences élaborées à la fois sur les activités de mesurage et sur le traitement et le sens de la mesure.

Notons qu'on retrouve des prescriptions très proches dans d'autres pays, par exemple dans les standards NCTM aux Etats Unis, même si les différentes notions ne sont pas introduites exactement aux mêmes niveaux. 


\section{Au lycée} filière scientifique. En seconde (MEN, 2010), lorsqu'ils mettent en œuvre des démarches expérimentales, les élèves doivent pouvoir « réaliser et analyser les mesures, en estimer la précision et écrire les résultats de façon adaptée ». Le programme de première souligne le rôle fondamental des activités expérimentales dans l'enseignement de la physique et de la chimie et insiste sur le fait que lors de ces activités les questions de mesure et d'incertitude sont cruciales : «Elles établissent un rapport critique avec le monde réel [...] où les mesures - toujours entachées d'erreurs aléatoires ou systématiques - ne permettent de déterminer des valeurs de grandeurs qu'avec une incertitude qu'il faut pouvoir évaluer au mieux ». On trouve dans ces programmes des références au rôle fondamental de la précision des mesures, à la fois dans l'enseignement et pour le progrès scientifique. Ces programmes citent des exemples issus de l'histoire des sciences dans lesquels l'augmentation du degré de précision des observations a permis d'établir de nouvelles lois. Ils indiquent que " l'automatisation de l'acquisition et du traitement des données expérimentales peut ainsi permettre de dégager du temps pour la réflexion, en l'ouvrant aux aspects statistiques de la mesure et au dialogue entre théorie et expérience». On voit ainsi apparaître dans ces nouveaux programmes des éléments d'épistémologie de la mesure.

En ce qui concerne la terminale, on trouve en annexe des programmes un document intitulé « Mesures et incertitudes » qui contient des indications destinées aux professeurs de sciences physiques et de mathématiques des lycées. Ce long document (une quarantaine de pages) présente la vision probabiliste de l'erreur développée par le BIPM. Il s'agit d'une clarification épistémologique à destination des enseignants. La liste des notions et des compétences expérimentales exigibles dans le programme de terminale est extrêmement développée et on citera par exemple :

- "Identifier les différentes sources d'erreur (de limites à la précision) lors d'une mesure : variabilités du phénomène et de l'acte de mesure (facteurs liés à l'opérateur, aux instruments,...).

- Évaluer et comparer les incertitudes associées à chaque source d'erreur.

- Expression et acceptabilité du résultat

- Exprimer le résultat d'une opération de mesure par une valeur issue éventuellement d'une moyenne, et une incertitude de mesure associée à un niveau de confiance.

- Déterminer les mesures à conserver en fonction d'un critère donné.

- Commenter le résultat d'une opération de mesure en le comparant à une valeur de référence. "

Pour pouvoir fournir aux élèves les outils nécessaires à l'évaluation des incertitudes sans qu'ils soient conduits à entrer dans le détail des outils mathématiques utilisés, les programmes suggèrent d'utiliser l'outil informatique.

71 Dans ces textes, les auteurs des programmes se positionnent d'un point de vue épistémologique sur une posture réaliste puisqu'ils postulent l'existence d'une valeur vraie de la grandeur. Cette valeur vraie (celle qu'on aurait obtenue avec une mesure parfaite) est inconnue, elle est même illusoire en raison de la variabilité des phénomènes, et on a donc accès uniquement à une valeur mesurée. Le résultat final de la mesure sera cette valeur, éventuellement issue d'une moyenne, assortie d'une incertitude résultant d'erreurs. Le programme différencie erreur et incertitude et conseille à l'enseignant de souligner cette distinction. Le terme de métrologie figure dans ces programmes qui affirment viser « une compréhension des bases de la métrologie » et qui préconisent de ce fait 
d'utiliser le lexique spécifique de la métrologie, tout en soulignant l'écart avec le vocabulaire courant.

La part consacrée dans les programmes à la question de la mesure et des incertitudes est donc croissante, avec une volonté affichée d'introduire dans les enseignements des sciences des éléments de métrologie et d'épistémologie.

\section{2. 3 Synthèse des recherches en didactique sur les questions liées à la mesure}

Dans plusieurs pays, des chercheurs se sont penchés sur les difficultés des étudiants sur la mesure. Ces recherches ont été motivées en partie par les difficultés des enseignants pour aborder la notion d'incertitude. Par exemple, chez les enseignants français de lycée, Séré et al. (1998) soulignent «une résistance certaine à aborder avec leurs élèves le problème des incertitudes » en partie par crainte que leurs étudiants deviennent sceptiques vis-à-vis de l'expérience, comme le souligne le bilan du projet européen «Labwork in Science Education » (Séré et al., 2001). Nous présentons dans la partie qui suit les résultats de certaines de ces recherches, concernant les conceptions des étudiants dans un premier temps, puis les propositions pour l'enseignement dans un second temps, avant de conclure sur cette synthèse des recherches antérieures.

\section{Les conceptions des élèves et étudiants sur la mesure}

De nombreux travaux, en particulier ceux de Séré, se sont penchés en France dans les années 90 sur les conceptions et les raisonnements des élèves et des étudiants. Ces recherches ont mis en évidence des difficultés liées au traitement des données et montré que les étudiants ont du mal à passer d'une vision déterministe de la science à une vision probabiliste, imposée par la dispersion des mesures, pourtant prescrite dans les instructions officielles (Séré, 1992). Les étudiants ont une notion de la mesure qui correspond à la recherche d'une "bonne" valeur en comparaison à une valeur de référence détenue par l'enseignant. Pour eux, les écarts correspondent à des erreurs de mesure, de manipulation (Séré et al., 1995). Le jugement effectué par l'étudiant à propos de la valeur se restreint donc à savoir s'il doit la garder ou l'éliminer (Séré et al., 1995). Dans un autre article, Séré montre que les étudiants effectuent une hiérarchie sur les mesures, la première étant la meilleure (Séré et al., 1993).

Le projet européen «Labwork in Science Education » portant sur les travaux pratiques dans l'enseignement des sciences en Europe a aussi questionné, entre autres, les difficultés des lycéens et des étudiants concernant la mesure. Cette recherche montre « $a$ lack of explicit understanding of the bases of estimating values from data sets » et le fait que " many students (between $30 \%$ et 60\%) appeared to think that with good enough apparatus and enough care it is possible to make a perfect measurement of a quantity ".

D'autres chercheurs (on citera par exemple Lubben et Millar, 1996, Allie et al., 1998; Lubben et al., 2001; Evangelinos et al., 2002; Lehrer, 2003, Volkwyn et al., 2004, 2008, Buffler et al., 2001, Buffler et al., 2009, Maisch et al., 2008, Maisch, 2010) ont cherché à caractériser les raisonnements liés à la mesure et aux incertitudes en physique.

Lubben et al. (1996) puis Buffler et al. (2001) ont catégorisé les raisonnements mis en œuvre par des élèves et des étudiants lorsqu'ils recueillent des données (nombre de valeurs à recueillir...), puis lors du traitement de ces données. Ces catégorisations ont été 
reprises par Volkwyn et al. (2004) qui identifient chez les étudiants trois types de raisonnement :

- raisonnement « point » : pour l'étudiant, il existe une « vraie valeur » (donnée par la littérature ou détenue par le professeur), une mesure qui est différente de cette bonne valeur est fausse, signe d'une erreur au sens péjoratif du terme. On peut accéder à cette " vraie valeur » en réduisant à zéro les erreurs dues au système de mesurage. Une seule mesure est alors considérée comme suffisante et le résultat est donné sous forme d'une valeur unique.

- raisonnement « ensemble » : l'étudiant entrevoit que toutes les mesures sont entachées d'incertitudes et qu'un grand ensemble de données est nécessaire pour pouvoir réaliser une étude statistique donnant une bonne approximation du mesurande et un intervalle de confiance. Le résultat de mesure s'exprime sous forme d'une valeur accompagnée d'un intervalle dans lequel se situe probablement cette valeur.

- raisonnement « mixte » : l'étudiant a conscience que la valeur de référence n'est pas possible à atteindre car il existe toujours des erreurs de manipulation, cependant il pense que cette valeur est la « bonne » valeur et il cherche à obtenir une mesure de la grandeur la plus proche possible de cette valeur de référence.

Cette catégorisation a été utilisée dans différentes recherches, par exemple pour évaluer l'impact de séances de travaux pratiques sur les raisonnements d'étudiants pour les non physics majors (Buffler et al., 2001) ou physics majors (Volkwyn et al., 2008).

Dans un article récent, Maisch et al. (2008) reprennent et affinent ces critères de catégorisation et les utilisent pour analyser les raisonnements d'étudiants français de première année universitaire dans différents contextes. Ces auteurs mettent en évidence le caractère situé des raisonnements des étudiants, montrant qu'ils dépendent du contexte (TP/TD). Dans sa thèse, Maisch (2010) a en outre montré que les étudiants n'utilisent quasiment jamais un raisonnement comportant une vision probabiliste de l'acte de mesurage.

Evangelinos et al. (2002) se sont penchés pour leur part sur l'interprétation par les étudiants d'une valeur singulière de mesure. Ils ont classé les explications des étudiants selon trois schèmes de raisonnement :

- exact : vision naïve d'un scientifique qui fait des mesures en utilisant un instrument de haute précision lui permettant d'obtenir la «valeur vraie»;

- approché : approche pragmatique selon laquelle la détermination de la « vraie valeur » n'est pas réalisable pour des raisons pratiques ;

- par intervalle : un résultat est présenté sous la forme d'un intervalle s'il a pour but de présenter le résultat d'une expérience réussie ou d'assurer la confiance dans le résultat.

81 Ces auteurs montrent que la majorité des étudiants ont des idées très éloignées du modèle scientifique généralement accepté (modèle probabiliste).

Buffler et al. (2009), pour leur part, en s'appuyant sur diverses études, définissent deux profils caractéristiques d'étudiants de première année d'université concernant leur image de la science et celle du sens de la mesure, et montrent le lien entre les deux. Pour certains étudiants, la nature a ses propres lois et l'expérimentation sert à les découvrir, le mesurage permettant d'obtenir des «valeurs vraies». Cette posture se rapproche d'un réalisme naïf. Pour d'autres, les théories scientifiques sont des inventions construites par les scientifiques à l'aide de leurs observations, la mesure fournissant des " preuves » par nature incertaines. On est ici plus proche du réalisme scientifique. Ces auteurs 
considèrent que " an explicit exposure to issue around the NOS (Nature of Science) in laboratory work may help in developing the required understanding of the relationship between scientific claims and experimental data ». On voit ici apparaitre la nécessité d'une articulation entre épistémologie et didactique lorsqu'on aborde les questions liées à la mesure dans l'enseignement.

\section{Des propositions pour l'enseignement}

statistiques des valeurs, «essayer de faire sentir intuitivement que toutes les valeurs, si elles ont été obtenues de la même façon, sont porteuses d'information » (Journeaux et al., 1995), expliciter et travailler sur les notions de précision et d'exactitude, développer le fait que la qualité de la mesure dépend de la question posée et donc de la décision à prendre. Dans le bilan du projet "Labwork in Science Education", Séré et al. (2001) regrettent que "les savoirs épistémologiques ne [soient] pas enseignés dans la plupart des pays et dans ceux où ils le sont, ce n'est pas à l'aide des TP ", alors que ces TP « offriraient de nombreuses occasions de traiter les liens complexes de la théorie et des données. ». Ils soulignent la richesse des démarches expérimentales du point de vue épistémologique, et pour ces auteurs " l'interaction entre le recueil de données (observation ou mesurage) et les savoirs théoriques sousjacents devrait être constante pendant des TP ». Ils considèrent que «le traitement des données et les conclusions qui en découlent, peuvent participer à des acquisitions autant conceptuelles qu'épistémologiques. ", citant comme exemple l'idée de confiance que l'on peut accorder aux données. Ils insistent aussi sur la nécessité d'assigner des objectifs clairs aux activités de mesurage. Leurs propositions sont basées sur l'idée d'utiliser les séances de TP pour introduire des savoirs d'ordre épistémologique, idée que l'on retrouve chez Buffler et al. (2009).

Evangelinos et al. (2002) et Buffler et al. (2008) insistent pour leur part sur la nécessité de situer l'apprentissage de la mesure dans un cadre probabiliste, et non fréquentiste comme c'est souvent le cas. Dans le cadre fréquentiste, on opère une description statistique de grands ensembles de données, mais l'analyse utilise des procédures liées aux erreurs aléatoires et des outils mathématiques tels que la moyenne. Le cadre probabiliste, au contraire, utilise les probabilités pour le traitement des données, l'ensemble des données étant représentées sous la forme de fonctions de densité de probabilité. Cette approche est en accord avec l'approche "incertitude" de la métrologie et est préconisée par l'organisation internationale pour la standardisation (international organization for standardization) dans leur publication Guide to the expression of uncertainty in measurement (GUM). Plusieurs groupes de chercheurs ont montré l'efficacité de l'utilisation du cadre probabiliste dans l'enseignement : «By introducing the concepts of probability and uncertainty as early as possible in the laboratory course, the tentative, yet quantifiable, nature of scientific knowledge is forgrounded [...] » (Pillay et al., 2008, p. 658). Ces études insistent également, comme Séré et al. (2001), sur la nécessité d'activités pratiques pour aider les étudiants à mieux comprendre l'activité de mesurage et les connaissances qui y sont liées. 

grandeur, en CM1 et CM2. Ces auteurs ont montré que lors de l'exploitation de ces séries de mesures les élèves sont capables d'envisager les trois causes possibles d'incertitudes (la grandeur à mesurer, l'instrument et l'expérimentateur), mais que l'expérimentateur est toujours, quelle que soit la grandeur, la cause d'erreur la plus citée par les élèves. Suivant l'instrument de mesure utilisé, les élèves mettent en cause cet instrument ou non, certains instruments, comme la balance électronique notamment, leur paraissant « infaillibles ». Concernant l'organisation et la gestion des données, la plupart des élèves sont capables de construire et d'analyser un tableau de fréquence et un diagramme en bâtons à partir de la liste de $\mathrm{N}$ mesures d'une même grandeur en prenant en compte l'ensemble des mesures de la classe. Certains d'entre eux sont même capables de raisonner en termes d'intervalle de confiance, en mobilisant un raisonnement qualitatif correct. Il est donc possible d'aller au-delà d'une approche fréquentiste dès l'école élémentaire et de commencer à utiliser, de façon qualitative, le cadre probabiliste pour l'interprétation des données. Munier et al. (2012) ont aussi montré que de jeunes élèves peuvent prendre conscience de la nécessité de répéter les mesures. Bien sûr, dans la plupart des cas, l'utilisation que l'on va faire des résultats de mesure ne nécessite pas de répéter de nombreuses fois ces mesures et il n'est pas question de préconiser un travail systématique de ce type pour chaque activité de mesurage pratiquée en sciences. D’une part, ceci est totalement irréaliste au vu notamment des contraintes de temps. D'autre part, cela n'est pas pertinent dans tous les cas. En revanche, une analyse statistique d'un grand nombre de mesures de la même grandeur est indispensable par exemple lorsqu'il s'agit de comparer les performances de deux instruments de mesure, de deux objets techniques ou la pertinence de deux méthodes de mesure. Il nous semble donc important, comme Journeaux et al. (1995) l'évoquent pour le lycée, de confronter les élèves à des situations dans lesquelles la prise de décision n'est possible qu'avec un grand nombre de mesures. À l'école élémentaire et jusqu'au lycée, on ne dispose pas encore des nombreux outils statistiques, développés en particulier en métrologie, permettant de traiter les résultats de mesure et l'âge des élèves impose un traitement simplifié des résultats de mesure. Nous venons de voir qu'à l'école on peut développer chez les élèves des raisonnements statistiques complexes, même s'il ne s'agit que de raisonnements qualitatifs. Au lycée, on pourra, comme le suggèrent les programmes, utiliser l'outil informatique pour le traitement statistique des données, tout en restant prudent sur l'interprétation que peuvent faire les élèves de ces données informatiques s'ils ne maîtrisent pas les outils mathématiques qui permettent de leur donner du sens. Séré et al. soulignent d'ailleurs que si l'ordinateur ainsi que les capteurs sont des moyens puissants de gagner du temps lors des mesurages « il faut alors leur donner tout leur sens et ne pas 
laisser les étudiants se retrancher derrière des routines sans signification » (Séré et al., 2001).

\section{Discussion sur cette synthèse de la littérature}

88 Ces différents résultats de recherche nous amènent à préconiser, comme dans la partie III.1, l'introduction d'éléments de métrologie dans les cursus scolaires. En particulier, un travail sur les instruments et sur certaines de leurs qualités pourrait aider les élèves à envisager qu'un instrument, quel qu'il soit, engendre des incertitudes, même une balance à affichage numérique. En effet, l'étude du principe d'un instrument de mesure peut permettre d'en comprendre les limites: par exemple pour les balances à ressort on suppose que le ressort est parfaitement élastique, pour un thermomètre à dilatation que la dilatation du verre est négligeable par rapport à celle du liquide. Ces phénomènes ne sont pas accessibles à l'école élémentaire, en revanche on peut les aborder au collège ou au lycée. Nous considérons qu'il serait pertinent, chaque fois que c'est possible, d'expliciter le principe de fonctionnement des instruments de mesure que rencontrent les élèves en soulignant leurs limites. À l'école élémentaire, peu d'instruments sont suffisamment simples pour se prêter à ce type d'étude, mais à cet âge on peut considérer que l'objectif essentiel est d'encourager les élèves à comparer divers instruments et à se doter d'outils permettant de choisir le plus performant dans une situation donnée.

Comme nous l'avons vu, une réflexion sur l'incertitude associée à tout processus de mesurage est fondamentale pour le physicien mais elle l'est aussi pour la formation du citoyen. Dans nos sociétés actuelles, tout individu est assailli de données numériques, de graphiques, qu'il s'agisse de vanter les propriétés d'un produit dans une publicité, de la présentation du bulletin météo, ou de défendre des choix économiques, politiques... Un des objectifs de l'enseignement est de doter les élèves d'outils cognitifs leur permettant d'analyser de façon critique toutes ces données. Cela passe entre autres par la prise en compte de la notion d'incertitude et du taux de confiance associé. Les différentes recherches que nous venons de citer montrent que les difficultés des élèves et étudiants sont importantes, mais qu'on peut initier les élèves dès le plus jeune âge à cette réflexion, et de telles approches nous semblent à développer étant donné l'importance de l'enjeu de société. Cependant, toutes ces recherches pointent que l'étude des questions de variabilité de la mesure et la prise en compte des incertitudes liées au mesurage a une place réduite dans les pratiques des enseignants, malgré leur importance dans les programmes, et que de ce fait les élèves ne disposent que de très peu des outils conceptuels permettant de raisonner sur la dispersion des résultats de mesure. En particulier, Séré et al. (1998) pointent que si les enseignants du lycée sont nombreux à calculer des moyennes dans des situations dûment repérées, si pour eux l'analyse des causes d'erreur est plus qu'un discours automatique qui suivrait une mesure, la " confiance qu'on peut avoir dans une mesure ", concept fondamental de l'approche statistique, est très largement absent ou jugé trop problématique pour être évoqué à ce niveau.

Bien que dès leur plus jeune âge les élèves manipulent des instruments de mesure et soient donc confrontés aux incertitudes liées au mesurage, et plus particulièrement à celles liées aux instruments de mesure, dans la pratique, les enseignants du primaire et $\mathrm{du}$ secondaire exploitent peu ou pas ces situations de mesurage et l'analyse des causes d'incertitude est souvent "court-circuitée ». On observe ici aussi, comme dans le cas de l'introduction des grandeurs à l'école, un décalage important entre les prescriptions et les pratiques des enseignants. On peut se demander si les conceptions des enseignants sur les 
concepts de grandeur et mesure ne font pas obstacle à la mise en œuvre des instructions officielles. Cette question a été étudiée par Passelaigue et al. (en cours de révision pour MTL) qui ont cherché à identifier les conceptions des futurs enseignants de l'école primaire et du secondaire concernant les concepts de grandeur et mesure.

\section{Les conceptions des enseignants}

\section{1 Concernant les grandeurs}

91 Quelle que soit leur posture épistémologique, nous avons vu que la distinction entre la notion de grandeur et celle de mesure est claire pour les scientifiques. Elle est aussi clairement affirmée dans les différents programmes scolaires qui distinguent, au moins dans les programmes du primaire, la phase d'identification des grandeurs, à travers des activités de comparaison d'objets, de la phase de mesurage nécessitant le recours à un étalon et à des instruments. Passelaigue et al. ont montré que cette distinction n'est pas évidente pour les enseignants ou futurs enseignants de l'école élémentaire qui ne différencient pas clairement ces deux concepts. Ces auteurs ont mis en évidence des difficultés conceptuelles importantes chez les professeurs des écoles stagiaires (PE2). Les concepts scientifiques de grandeur et mesure sont peu, voire pas du tout maitrisés par certains PE2, principalement le concept de grandeur. De plus, ces auteurs ont mis en évidence une conception erronée du concept de grandeur, bien éloignée de la définition scientifique. Pour une part importante des professeurs des écoles stagiaires, une grandeur correspond à quelque chose de flou, de mal déterminé, de peu précis. Ils semblent mobiliser les raisonnements suivants :

- Le objets ont des propriétés (ou qualités) : ils sont plus ou moins lourds, longs, chauds, etc ;

- Ces qualités permettent de les ranger, du plus léger au plus lourd, du plus court au plus long, etc et certains objets sont considérés comme ayant le même ordre de grandeur pour la qualité considérée (ils ont presque la même longueur, la même masse...);

- Pour les comparer ensuite précisément il est indispensable de mesurer la longueur, la masse, ... de ces objets.

La comparaison des grandeurs directement, et non la comparaison de leurs mesures, n'est pas envisagée, or c'est elle qui permet de définir la grandeur physique. En particulier, il semble que la grandeur soit considérée comme une qualité, « ce que l'on mesure », ce qui est correct, mais qu'avant sa mesure ce ne soit qu'une qualité approximative, comme si «qualité » s'opposait à " précision ». Seul le passage au quantitatif, donc au nombre, par le biais de la mesure, permettrait d'accéder à cette précision.

On pourrait penser que ces difficultés sont liées au fait que beaucoup de futurs enseignants $d u$ primaire n'ont pas une formation initiale scientifique suffisante. Cependant, une étude des définitions de "grandeur» et "mesure» données par des professeurs stagiaires de lycée et collège en mathématiques et sciences physiques montre que, même s'ils sont moins nombreux que les professeurs des écoles à considérer la grandeur comme quelque chose d'imprécis, par opposition à la mesure qui aurait l'apanage de la précision, cette conception erronée de grandeur apparait tout de même chez certains d'entre eux. Cela montre qu'un cursus universitaire scientifique ne suffit pas pour dépasser cette conception. Malgré leur formation universitaire en sciences au moins jusqu'au niveau L3, les futurs enseignants de mathématiques et de sciences physiques éprouvent, comme les enseignants du premier degré, beaucoup de difficultés à 
définir le concept de "grandeur ». Notons que c'est le concept de grandeur qui pose le plus de problèmes aux enseignants et qui semble le moins maîtrisé.

On peut penser que ces difficultés des enseignants à définir et dissocier les concepts de grandeur et mesure sont à l'origine des pratiques couramment observées dans les classes. En effet, pour que les enseignants soient en mesure de s'approprier les enjeux des prescriptions et de les mettre en œuvre, ils doivent a minima maîtriser ces concepts et être capables de les différencier, ce qui n'est manifestement pas le cas chez les enseignants stagiaires interrogés par Passelaigue et al. Comment s'étonner alors du fait que certains enseignants ne passent pas par l'étape de comparaison des grandeurs, ou que cette phase soit conduite de façon formelle et très rapide, comme c'est souvent le cas dans les manuels. Qu'en est-il alors des bénéfices de cette phase pour les élèves?

\section{2 Concernant les incertitudes liées au mesurage}

Peu d'études se sont penchées sur les conceptions des enseignants concernant la mesure. Dans une étude exploratoire auprès de plus de 200 PE2, Passelaigue et al. ont effectué un recueil de mots pour avoir une première idée de ce que chacun des termes "grandeur » et «mesure » évoque spontanément pour les futurs enseignants de l'école élémentaire. L'analyse des réponses met en évidence, pour le terme mesure, une absence totale de propositions sur la précision et les incertitudes de mesure. Une recherche plus poussée effectuée auprès de 91 PE2 a montré que pour une part très importante des stagiaires interrogés, les termes de précision et d'incertitude se rapportent davantage au concept de grandeur qu'à celui de mesure, alors que du point de vue scientifique ces termes se réfèrent pourtant sans aucune ambiguïté à la mesure et au mesurage. Pour certains, cela semble lié à leurs conceptions erronées de la grandeur : si la grandeur est imprécise, la mesure ayant l'apanage de la précision, associer mesure à incertitude n'a pas de sens, et on trouve des justifications comme "On n'est plus dans l'incertitude grâce à une mesure", " La grandeur ne peut pas être certaine mais la mesure oui ».

Dans le second degré, Séré et al. (1998) ont montré que si les enseignants de sciences physiques déclarent éprouver une réticence certaine à aborder avec leurs élèves le problème des incertitudes («Parler des incertitudes ne serait pas pour eux un but en soi »), ils abordent en fait le problème mais avec un vocabulaire plus flou et adapté aux situations, et avec des arguments variés. Les enseignants ont majoritairement en tête un traitement statistique des mesures, mais il peut rarement être mené de bout en bout. Ces auteurs soulignent que sur ce sujet délicat la pratique enseignante a du mal à se stabiliser et laisse beaucoup de place à l'initiative personnelle.

\section{Discussion}

97 Nous avons montré dans les parties II et III la difficulté des concepts de grandeur et mesure, la diversité des postures épistémologiques permettant de les aborder et le flou dans lequel les enseignants sont laissés pour aborder ces questions avec les élèves. Nous avons en effet mis en évidence que les programmes français ne se positionnent pas explicitement $\mathrm{du}$ point de vue épistémologique et que, de plus, la posture épistémologique des concepteurs de programmes semble différente dans l'enseignement primaire et dans l'enseignement secondaire, ou suivant les grandeurs considérées. Nous avons souligné à plusieurs reprises l'intérêt de développer dans l'enseignement une 
approche épistémologique des grandeurs et mesures et d'introduire, serait-ce de façon indirecte, sans en nier les obstacles ni les difficultés, des éléments de métrologie dans les cursus scolaire, et ce dès l'école élémentaire. Or les résultats présentés dans les parties III et IV concernant les enseignants montrent qu'ils ne sont pas en mesure de s'approprier les enjeux des prescriptions officielles concernant l'introduction des grandeurs et qu'ils sont parfois démunis pour aborder les questions liées à la mesure et aux incertitudes avec leurs élèves.

\section{Quelle formation pour les enseignants ?}

Toutes ces analyses montrent qu'il est nécessaire de développer la formation des enseignants dans le domaine des grandeurs et mesures, à la fois pour augmenter leur niveau de maîtrise des concepts en jeu, pour qu'ils soient en mesure de mettre en œuvre les prescriptions officielles et d'avoir du recul sur les enjeux de ces prescriptions qui, nous l'avons vu, sont très pointues sur ces questions. Il y a, d'une part, un besoin de formation épistémologique concernant ces concepts et, d'autre part, un besoin de formation didactique pour réfléchir sur la façon dont on peut les travailler avec les élèves.

Concernant le volet épistémologie, les enseignants ne sont pas au clair sur la définition des concepts de grandeur et mesure et sur la manière dont les grandeurs sont construites. Ils différentient mal ces deux concepts qui sont pour beaucoup "amalgamés». Une réflexion épistémologique sur ces concepts, sur leur histoire et sur les courants épistémologiques, opérationnalisme et réalisme, qui se penchent sur leur signification et sur leurs liens à la réalité et à la théorie, permettrait de travailler avec eux sur ces concepts. Ce travail pourrait permettre de leur faire prendre conscience de leur propre posture épistémologique et des positions des concepteurs des programmes. On peut d'ailleurs penser que si dans les programmes ces dernières étaient davantage explicites, cela faciliterait le travail des enseignants qui peuvent se trouver démunis pour aborder ces questions avec leurs élèves, à l'école comme dans l'enseignement secondaire. La formation épistémologique des enseignants devrait en outre intégrer des éléments sur l'histoire et l'épistémologie de la mesure et des éléments de métrologie. Nous avons vu que l'annexe des nouveaux programmes de terminale laisse penser que les concepteurs sont conscients des lacunes qui existent dans la formation des enseignants sur le traitement des données de mesure et les incertitudes. On peut toutefois craindre que la diffusion d'un document sur le site institutionnel eduscol n'ait pas le même impact qu'une réelle formation initiale et continue. Si on veut en outre que les enseignants puissent introduire des éléments d'épistémologie et de métrologie dans leurs enseignements comme préconisé par plusieurs des auteurs cités dans les parties précédentes, il faut non seulement que les enseignants aient cette culture épistémologique mais aussi qu'ils disposent d'éléments didactiques concernant la transmission de ces savoirs.

Nous l'avons souligné à plusieurs reprises, il y a un enjeu fort à travailler avec les élèves sur les concepts de grandeur et mesure, sur la notion d'incertitude de mesure, sur l'instrumentation, dès l'école élémentaire. Séré et al. (2001) insistent aussi sur l'importance de ce type de travail dans le secondaire dans le bilan du projet européen "Labwork in Science Education» et préconisent de développer l'enseignement des savoirs épistémologiques. Or comme le disent Séré et al. (1998): "la situation des enseignants n'est pas confortable [...] Comment faire cohabiter les théories bien établies qui lui 
donnent un air de vérité, et les expériences qui mettent en jeu les imperfections des appareils et des manipulations?». Ces auteurs considèrent qu'à l'heure actuelle "les enseignants peuvent "dire" une attitude raisonnable, différenciée d'une situation à l'autre, et exprimer ce qu'il faudrait "faire", en se gardant cependant de le faire par manque de temps et aussi des concepts indispensables ». Il est donc fondamental de développer aussi la formation didactique des enseignants.

101 Même si les recherches dans ce domaine ne sont pas très nombreuses, elles ont tout de même permis de construire des situations de classe qui permettent de travailler avec les élèves sur le sens de la mesure et de jeter les premiers jalons d'un authentique traitement des mesures. Des ingénieries existent pour cela et pourraient être utilisées en formation. On peut citer par exemple le travail de Passelaigue (2011), qui montre tout l'intérêt de travailler sur la grandeur masse avec des activités de comparaison directe avant d'introduire la mesure. On peut penser que la diffusion de ces résultats pourrait montrer aux enseignants qui ne sont pas convaincus par cette approche tout son intérêt. Citons aussi les travaux de Maisch (2010) et Munier et al., qui montrent des situations efficaces permettant de travailler avec les élèves d'âges très différents sur les causes d'erreurs et le traitement des mesures. Il reste encore beaucoup de travail pour élaborer, à partir des recherches menées avec les élèves, des formations pour les enseignants. En particulier, il faudra développer des analyses de pratiques enseignantes dans ce domaine, notamment sur la posture des enseignants: Se positionnent-ils par rapport aux deux courants épistémologiques? Si oui comment? Leur posture est-elle explicite? A-t-elle une influence sur leur façon d'aborder les grandeurs, les activités de mesurage et le traitement des mesures? Nous avons encore besoin de mieux comprendre ce qui se joue à l'intérieur de la classe sur ces questions pour pouvoir développer des ingénieries de 
formation pour les enseignants, mais cela nous semble un objectif essentiel pour les recherches en didactique des sciences à l'aube du XXIe siècle.

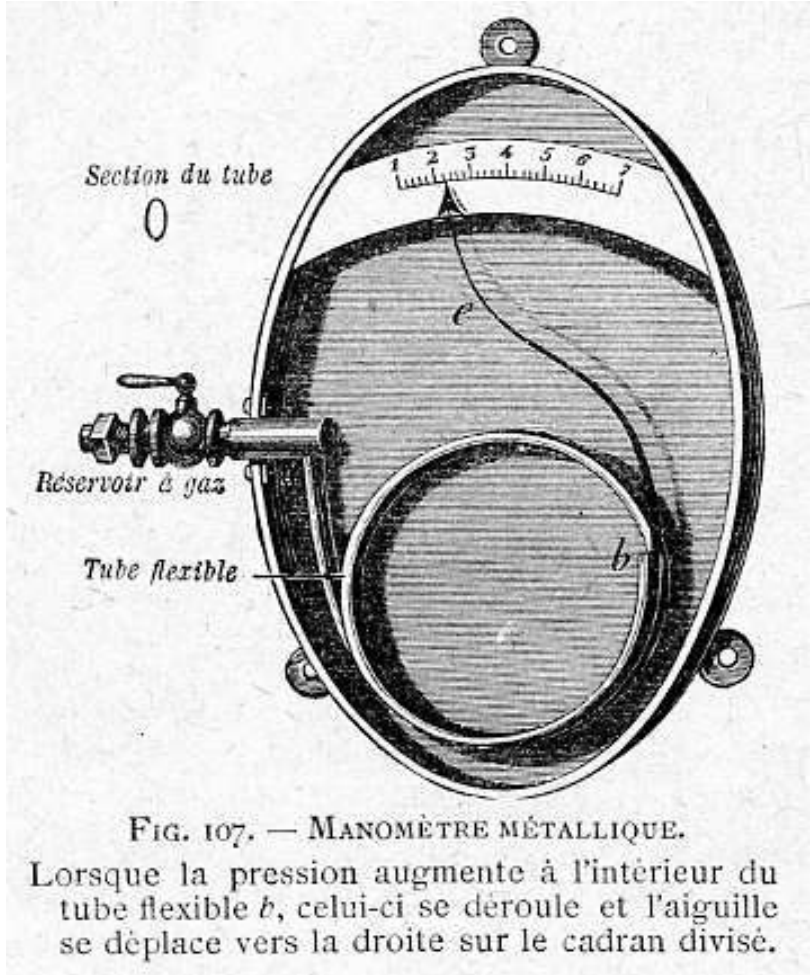

Cours abrégé de physique : école primaires supérieures, cours complémentaires, préparation au brevet élémentaire (Programmes de 1920). Chassagny, M., Carré, F., Hachette, Paris, 1922. Source CEDRHE, 22941, p. 136

\section{BIBLIOGRAPHIE}

Allie, S., Buffler, A., Kaunda, L., Campbell, B. and Lubben, F., First-year physics students' perceptions of the quality of experimental measurements, International Journal of Science Education , 20 (4), 1998, p. 447-459.

BIPM (Bureau International des Poids et Mesures). Vocabulaire international de métrologie, JCGM. 2008.

Boulton-Lewis, G. M., Wilss, L. A., and Mutch, S. L., An analysis of young children's strategies and use of devices of length measurement, Journal of Mathematical Behavior, 15, 1996, p. 329-347.

Brousseau, G. et Brousseau, N., Le poids d'un récipient, étude des problèmes de mesurage en CM, Grand N, 50, 1991-1992, p. 65-87.

Buffler, A., Allie, S., Lubben, F., The development of first year physics students' ideas about measurement in terms of point and set paradigms, International Journal of Science Education, 23 (11), 2001, p. 1137-1156. 
Buffler, A., Allie, S., Lubben, F., Teaching measurement and uncertainly the GUM way, The Physics Teacher, 46, 2008, p. 539-543.

Buffler, A., Lubben, F., and Ibrahim, B., The relationship between student's viewsof the nature of science and their views of the nature of scientific measurement, International Journal of Science Education, 31 (9), 2009, p. 1137-1156.

Bureau International des Poids et Mesures. JCGM-VIM. [International Vocabulary of Metrology Basic and general concepts and associated terms] <http://www.bipm.org/utils/common/documents/jcgm/JCGM_200_2008.pdf>. 2008

Carnap, R., Les fondements philosophiques de la physique (tr. fr.). Paris : Armand Colin. 1973.

Chalmers, A. F., Qu'est ce que la science? Récents développements en philosophie des sciences: Popper, Kuhn, Lakatos, Feyrabend (tr. fr.). Paris : Editions La découverte. 1987.

Chambris, C., Relations entre les grandeurs et les nombres dans les mathématiques de l'école primaire. Evolution de l'enseignement au cours du XXe siècle. Connaissance des élèves actuels. Thèse de didactique des mathématiques. Université Paris 7, Paris. 2008.

Chevallard, Y. et Bosch, M., Les grandeurs en mathématiques au collège. Partie i. une Atlantide oubliée, Petit $X, 55,2000-2001$, p. 5-32.

Clements, D. H., and Stephan, M., Measurement in pre-K to grade 2 mathematics. Dans D. H. Clements and J. Sarama (Eds.), Engaging young children in mathematics: Standards for early childhood mathematics education. Mahwah, NJ : Lawrence Erlbaum Associates. 2004, p. 299-320.

Coelho, S., Contribution à l'étude didactique du mesurage en physique dans l'enseignement secondaire. Thèse de didactique des disciplines, Université Paris 7, Paris. 1993.

Cotteret, M.-A., Métrologie et enseignement. Doctoral dissertation in education Science. Université Paris 8-Vincennes-Saint-Denis, Paris. 2003.

Dagognet, F., Réflexions sur la mesure. La Versanne : Editions encre marine. 1993.

De Broglie, L., Société Nouvelle de l'Encyclopédie Française, Tome II. Paris : Larousse. 1955.

D’Espagnat, B., Le réel voilé. Domont : Librairie Arthème Fayard. 1994.

Diénes, Z. P. et Golding, E. W., Les premiers pas en mathématiques : exploration de l'espace et pratique de la mesure ( $5^{\circ}$ edition). Paris : OCDL. 1970.

Douady R. et Perrin-Glorian M. J., Un processus d'apprentissage du concept d'aire de surface plane, Educational Studies in Mathematics, 20, 1989, p. 387-424.

Esfeld, M., Philosophie des sciences, une introduction. Lausanne : Presses polytechniques et Universitaires romandes. 2006.

Encyclopédie Universalis. 1993, 2008.

Evangelinos, D., Psillos, D. and Valassiades, O., An Investigation of Teaching and Learning about Measurement Data and their Treatment in the Introductory Physics Laboratory. Dans D. Psillos and H. Niederer (eds.), Teaching and Learning in the Science Laboratory. Dordrecht : Kluwer Academic. 2002, p. 179-190.

Favrat J. F. et Munier, V., Evolution de l'enseignement des grandeurs à l'école élémentaire depuis 1945, Repères IREM, 68, 2007, p. 51-75.

Friedelmeyer, J. P., Grandeurs et nombres : l'histoire édifiante d'un couple fécond, Repères IREM, 44, 2001, p. 5-31. 
Guay, A., Appareil image et particule. Dans J. L., Deotte, M. Froger, et S. Mariniello, Appareil et intermedialité. Paris : Editions de l'Harmattan. 2007, p. 97-120.

Hacking, I., Representing and intervening. Cambridge: Cambridge University Press. 1983.

Hiebert, J., Why do some children have trouble learning measurement concepts ? Arithmetic Teacher, March, 3 (7), 1984, p. 19-24.

Journeaux, R., Séré, M.G. et Winther, J., La mesure en terminale scientifique, Bulletin de l'Union des Physiciens, 779 (1), 1995, p. 1925-1945.

Kospentaris, G., Spyrou, P. and Lappas, D., Exploring students' strategies in area conservation geometrical tasks, Educational Studies in Mathematics, 77, 2011, p. 105-127.

Kuhn, T. S., La tension essentielle (tr. fr.). Paris : Gallimard. 1977, 1990.

Langlois, F. et Munier, V., Les valeurs efficaces en électricité : une perte de sens à travers l'évolution des programmes, Bulletin de l'union des physiciens, 105, 2011, p. 743-763.

Lascours, J. et Calmettes, B., Difficultés d'étudiants à propos des circuits en courant alternatif, Bulletin de l'Union des Physiciens, 800, 1998, p. 41-61.

Le Chatelier, R. H., La Silice et les silicates. Site Internet de la Bibliothèque Nationale de France. Disponible sur internet : <http//gallica.bnf.fr $>1994$.

Lehrer, R., Schauble, L., Strom, D. and Pligge, M., Similarity of form and substance: Modeling material kind. Dans D. Klahr et S. Carver (eds.), Cognition and Instruction: 25 years of progress. Mahwah, N. J. : Lawrence Eribaum Associates, Inc. 2001, p. 39-74.

Lehrer, R., Developing understanding of measurement. Dans J. Kipatrick, W.G. Martin, and D.E. Schifter (eds.), A research companion to principles and standards for school mathematics. Reston, VA: National Council of Teachers of Mathematics. 2003, p. 197-192.

Lippmann, R., Teaching the concepts of measurement: an example of a concept-based laboratory course, American Journal of Physics, 73 (8), 2005, p. 771-777.

Lubben, F. and Millar, R., Children's ideas about the reliability of experimental data, International Journal of Science Education, 18 (8), 1996, p. 955-968.

Lubben, F., Campbell, B., Buffler, A. and Allie, S., Point and Set Reasoning in Practical Science Measurement by Entering University Freshmen, Science Education, 85 (4), 2001, p. 311-327.

Macdonald, A., Young children's representations of their developing measurement understandings. Dans J. Clark, B. Kissane, J. Mousley, T. Spencer and S. Thornton (eds), Proceedings of the 34th annual conference of the Mathematics Education Research Group of Australasia and the Australian Association of Mathematics Teachers. Adelaide, Australia. 2011, p. 482-490.

Maisch, C., Ney, M. et Balacheff, N., Quelle est l'influence du contexte sur les raisonnements d'étudiants sur la mesure en physique ? Aster, 47, 2008, p. 43-70.

Maisch, C., Étude des raisonnements d'étudiants sur la mesure en TP de physique de première année universitaire : influence du contexte et effet de rétroaction. Thèse de didactique, Université Joseph Fourier, Grenoble. 2010.

Malifaud, P., Mesure. In Encyclopædia Universalis. Paris : Enclycopædia Universalis.

Disponible sur Internet : <http://www.universalis-edu.com>. 2008.

Mäntylä, T., and Koponen, I., Understanding the role of measurements in creating physical quantities : A case study of learning to quantify temperature in physics teacher education, Science and Education, 16, 2006, p. 291-311. 
Maruani, A., Aspects de la mesure; repères, problématiques et enjeux, Bulletin de l'Union des Physiciens, 787/90, 1996, p. 1433-1443.

Merle, H., et Munier, V., Comment conceptualiser la hauteur du Soleil en tant qu'angle au cycle 3 ? Aster, 36, 2003, p. 39-68.

Ministère de l'Education Nationale (France). Documents d'application des programmes de 2002, grandeurs et mesures à l'école élémentaire. Paris : direction de l'enseignement scolaire. 2002.

Ministère de l'Education Nationale, de l'enseignement supérieur et de la recherche (France). Socle commun de connaissances et de compétences et modifiant le code de l'éducation. 11 juillet. 2006.

Ministère de l'Education Nationale (France). Programmes d'enseignement de l'école primaire, Bulletin officiel de l'éducation nationale, hors série $n^{\circ} 3$ du 19 juin. 2008a

Ministère de l'éducation Nationale (France). Programmes du collège, Bulletin officiel spécial $n^{\circ} 6$ du 28 août 2008. 2008b.

Ministère de l'éducation nationale (France). Bulletin officiel spécial n 4 du 29 avril 2010. 2010.

Ministry of Education (New Zealand). Geometry and measurement. Disponible sur Internet : < http://nzmaths.co.nz/node/1277>. 2009.

Munier, V., Merle, H. et Brehelin, D., Measurement and Uncertainty in Elementary School : Collecting, Organizing, Representing, and Interpreting Measurements, à paraître dans International Journal of Science Education.

Nadi, M., Grandeur de la mesure, Bulletin de l'Association des Professeurs de Mathématiques de l'Enseignement Public, 428, 2000, p. 292-300.

National Council of Teachers of Mathematics., Principles and Standards for School Mathematics. Reston, VA : Author. 2000.

Passelaigue, D., Grandeurs et mesures à l'école élémentaire. Des activités de comparaison a la construction des concepts : le cas de la masse en CE1. Thèse de didactique des sciences physiques. Université Montpellier 2, Montpellier. 2011.

Passelaigue, D. and Munier, V., Schoolteachers' Difficulties about the Concepts of Quantity and Measurement, soumis à Mathematical Thinking and learning.

Paty, M., Instruments de mesure. Philosophies de la mesure. Dans J.C. Beaune (ed.), La mesure, instruments et philosophies. Seyssel : Champ Vallon. 1994, p. 159-174.

Perdijon, J., La mesure, sciences et philosophie. Paris : Flammarion. 1998.

Perdijon, J., La mesure, histoire, sciences et technique. Paris: Vuibert. 2012.

Perrin-Glorian, M. J., Problèmes didactiques liés à l'enseignement des grandeurs. Le cas des aires. Dans Dorier, J. L. et al. Actes de la 11e école d'été de didactique des mathématiques. Grenoble : La Pensée sauvage. 2002, p. 299-315.

Petrosino, A J., Lehrer, R. and Schauble, L., Structuring Error and Experimental Variation as Distribution in the Fourth Grade, Mathematical Thinking and Learning, 5 (2-3), 2003, p. 131-156.

Piaget, J. et Inhelder, B., Le développement des quantités physiques chez l'enfant. Neuchâtel et Paris : Delachaux et Niestlé. 1968.

Pillay, S., Buffler, A., Lubben, F. et Allie, S., Effectiveness of a GUM-compliant course for teaching measurement in the introductory physics laboratory, European Journal of Physics, 29, 2008, p. 647-659. 
Pressiat, A., Les grandeurs dans les mathématiques et dans leur enseignement Conférence pour l'Association des Professeurs de Mathématique de l'Enseignement Public, 483. Besançon. Disponible sur internet : <www.apmep.asso.fr>. 2009.

Quillet, A., Nouvelle encyclopédie autodidactique Quillet (ouvrage collectif). Paris : Librairie A. Quillet. 1961.

Robert, M., Khantine-Langlois, F., Munier, V. et Dusseau, J. M. Grandeurs et mesures en électrocinétique des courants variables, Aster, 47, 2008, p. 71-102.

Rouche, N., Nombres, grandeurs, proportions. Paris : Ellipses. 2006.

Sankey, H., Qu'est-ce que le réalisme scientifique ? Divinatio, 12, 2002, p. 103-120. Disponible sur internet : <http://philosophy.unimelb.edu.au/about/staff/Sankey/> traduit par Noelle Colombet Sankey.

Séré, M. G., Le déterminisme et le hasard dans la tête des élèves ou de l'utilité d'un traitement statistique des séries de mesures, Bulletin d'Union des Physiciens, 740, 1992, p. 87-96.

Séré, M. G., Journeaux, R., and Larcher, C., Learning the statistical analysis of measurement errors, International Journal of Science Education, 15(4), 1993, p. 427-438.

Séré, M. G., et Journeaux, R., Le traitement statistique des mesures en travaux pratiques de physique de DEUG : Une innovation à Orsay, Didaskalia, 6, 1995, p. 165-177.

Séré, M.G., Journeaux, R. et Winther, J., Enquête sur la pratique des enseignants de lycée dans le domaine des incertitudes, Bulletin d'Union des Physiciens, 801, 1998, p. 247-254.

Séré, M. G., Winther J., Le Maréchal J. F. et Tiberghien A., Le projet européen "Labwork in Science Education" [Les Travaux pratiques dans l'enseignement des sciences en Europe] Bilan et perspectives, Bulletin de l'Union des Physiciens, 839, 2001, p. 1727-1740.

Séré, M. G., La mesure dans Le Bup, Bulletin de l'Union des Physiciens, 898 (1), 2007, p. 44-47.

Séré, M. G., La mesure dans l'enseignement des sciences physiques : évolution au cours du temps, Aster, 47, 2008, p. 25-42.

Ullmo, J., La pensée scientifique moderne. Paris : Flammarion. 1969.

Vocabulaire International de la Métrologie, 1993 et 2008. Disponible sur internet : <http// www.bipm.org/utils/common/documents/jcgm/JCGM_200_2008.pdf>.

Volkwyn, T.S., Allie, S., Buffler, A., Lubben, A. and Campbell, B., First year physics students' understanding of the measurement in the context of laboratory practicals. Dans A. Buffler and R. C. Laugksch (eds.), Proceedings of the 12th Annual Conference of the South African Association for Research in Mathematics, Science and Technology Education. 2004, p. 1011-1017.

Volkwyn, T. S., Allie, S., Buffler, A. and Lubben, A., Impact of a conventional introductory laboratory course on the understanding of measurement, Physical Review Special Topics Physics Education Research, 4 (1), 2008, p. 10.

Worrall, J., Structural Realism : the Best of Both worlds, Dialectica, 43 (1-2), 1989, p. 99-124.

Zahar, E., Essai d'épistémologie réaliste (tr. fr.). Paris : Vrin. 2000.

\section{NOTES}

1. Notons que de ce fait nous excluons les « grandeurs » comme la gentillesse, etc. 


\section{RÉSUMÉS}

Dans cet article, nous nous intéressons à la question de l'articulation entre didactique et épistémologie dans l'enseignement des grandeurs et de la mesure. Dans un premier temps, nous discutons le sens de ces concepts ainsi que les différentes postures épistémologiques avec lesquelles on peut les aborder. Nous analysons ensuite la façon dont sont introduites les grandeurs dans l'enseignement en ciblant quelques exemples de grandeurs (grandeurs physiques à l'école et grandeurs électriques au collège). Nous étudions également la place que les programmes accordent à la question du traitement de la mesure et des incertitudes de mesure avant de présenter une synthèse des recherches en didactique dans ce domaine. Enfin, nous nous intéressons aux conceptions des enseignants et nous discutons la question de la formation des enseignants.

In this paper we question the links between science education and epistemology in the teaching of quantities and measurement. We first discuss the meaning of these concepts and the epistemological stances with which we can approach them. We then analyse the way quantities are introduced in school, focusing on some examples of quantities (physical quantities at elementary school and electric quantities at middle school). We also study the place devoted by the official instructions to the issue of the treatment of measurement data and uncertainties of measure, before presenting a synthesis of the researches in this domain. Finally we study the teachers' conceptions about quantities and measurements and discuss the question of teacher training.

\section{INDEX}

Mots-clés : collège, école élémentaire, épistémologie, grandeur, mesure

Keywords : attribute, elementary school, epistemology, measurement, middle school, quantity

\section{AUTEURS}

\section{VALÉRIE MUNIER}

LIRDEF (EA 3749), IUFM de l'académie de Montpellier - UM2 et Université Montpellier 3

\section{DOMINIQUE PASSELAIGUE}

LIRDEF (EA 3749), IUFM de l'académie de Montpellier - UM2 et Université Montpellier 3 